\title{
Effect of Seepage Flow on Sediment Incipient Motion around a Free Spanning Pipeline
}

Zhen Guo ${ }^{1}$, Dong-Sheng Jeng ${ }^{2}$, Hongyi Zhao ${ }^{3}$, Wei Guo ${ }^{4}$, Lizhong Wang ${ }^{5 *}$

${ }^{1}$ Key Laboratory of Offshore Geotechnics and Material of Zhejiang Province, Research Center of Coastal and Urban Geotechnical Engineering, College of Civil Engineering and Architecture, Zhejiang University, Hangzhou, 310058, China; E-mail: nehzoug@163.com

${ }^{2}$ Griffith School of Engineering, Griffith University Gold Coast Campus, Queensland, QLD 4222, Australia; Email: d.jeng@griffith.edu.au

${ }^{3}$ State Key Laboratory of Hydrology-Water Resources and Hydraulic Engineering, Hohai University, Nanjing, 210098, China; E-mail: zhaohongyi77@sina.com

${ }^{4}$ National Engineering Laboratory for High Speed Railway Construction, School of Civil Engineering, Central South University, Changsha, Hunan, 410075, China; E-mail: wei.guo.86@gmail.com

5* Corresponding author, Key Laboratory of Offshore Geotechnics and Material of Zhejiang

Province, Research Center of Coastal and Urban Geotechnical Engineering, College of Civil

Engineering and Architecture, Zhejiang University, Hangzhou, 310058, China; E-mail:

wlzzju@163.com. Tel.: +86571 88208678. Fax: +86 57188206240

\begin{abstract}
Sediment incipient motion is the first step of the whole process of sediment transport. However, previous numerical works simplified the seabed surface as a type of impermeable and rigid boundary, and ignored the effect of seepage flow on the mobility of bed particles. In this paper, to reveal the physics behind sediment incipient motion around a free spanning pipeline, an integrated numerical model, coupling the SST (Shear-Stress Transport) turbulence model with the porous seabed model, was proposed. Numerical studies showed that with the periodic formation and shedding of vortices around the pipeline, both the oscillatory and residual excess pore-pressures developed within the seabed. In some cases, the vertical gradient of excess pore-pressure (seepage force) had a significant impact on the mobility of bed particles around the pipeline. It was found that lower saturation degree and seabed permeability would remarkably increase both the oscillatory and residual seepage forces, and thus enhance bed particle mobility. While for a sandy seabed with smaller soil shear modulus, the oscillatory seepage force was only slightly reduced, but a high residual seepage force would be generated with time. This could lead to obvious decrease in the submerged weight of bed particles, making them more easily dragged away from the seabed.
\end{abstract}

Key words: free spanning pipeline; turbulent flow; seepage flow; sediment incipient motion; oscillatory pore-pressure; residual pore-pressure. 


\section{Introduction}

Submarine pipelines play a significant role in the exploitation of marine oil and gas. In the natural environments, free spans are likely to occur when the pipeline passes over areas with uneven seafloor, or when upheaval buckling occurs due to a constrained thermal expansion. This leads to flow turbulence and instability around the free spans, and induces local scour in the underlying seabed (Sumer et al., 2003), which would significantly threaten the structural safety of pipelines.

As the first step of local scour adjacent to pipelines, the incipient motion of sediment particles is affected by many factors, such as the near-seabed flow velocity, particle diameter and submerged weight. Considerable efforts have been devoted to determine the threshold condition for sediment incipient motion, among which the most widely cited work to date is the Shields diagram (Shields, 1936). The initial usage of the Shields diagram was only limited to the unidirectional flows and horizontal or near-horizontal bed slopes. As an extension, some modifications have been made to the traditional Shields diagram in order to make it suitable for more general conditions, such as oscillatory flows (Madsen and Grant, 1976) and bed slopes with an inclination angle (Whitehouse and Hardisty, 1988; Chiew and Parker, 1994).

In essence, the incipient motion of a bed particle is the result of the joint effect of the driving and the resistance forces. The driving force is horizontal or tangential to the bed surface and is generally linked with the bed shear stress (caused by near-seabed fluid flow). The resistance force is a type of Coulomb force that is proportional to the resultant force of vertical forces including submerged weight of sediment particle (particle weight in air minuses its buoyant force under hydrostatic conditions), flow-induced lift force, seepage force and so on. To simplify the analysis in previous researches, the seepage force is always ignored by assuming the seabed to be impermeable. However, for a seabed in the form of sand particles or gravels, the fluid flow could induce two kinds of seepage flows, which are named "suction" and "injection", across the upper boundary of the seabed. The suction tends to produce an additional downward seepage force, and the upward seepage force acts on the sediment particles for the condition of injection. As observed in numerous experiments (Oldenziel and Brink, 1974; Richardson et al., 1985; Cheng, 1997; Cheng and Chiew, 1999; Ramakrishna Rao and Nagaraj, 1999; Sumer et al., 2011; Sumer et al., 2013), the influence of suction or injection on the incipient motion of sediment particles is significant. Therefore, the assumption of seabed impermeability may be oversimplified and inappropriate.

To date, there have been many studies on the numerical simulations of local scour around 
submarine pipelines (Sumer and Fredsøe, 2002; Li and Cheng, 2001; Liang et al., 2005a, b). However, in these numerical studies, the seabed was simplified as an impermeable medium and the seepage effect on sediment incipient motion was ignored. Therefore, this paper is aimed to study the development of excess pore-pressures within the seabed around a free spanning pipeline. An integrated numerical model is proposed, in which the SST (ShearStress Transport) turbulence model is used to simulate the upper flow field, and a novel porous seabed model is adopted to predict the dynamic response of the underlying seabed. In the simulation, the fluid pressure calculated by the SST model was taken as the pressure boundary condition in the porous seabed model, and then the seabed responses, including excess pore-pressures, effective stresses and deformations of the soil skeleton, could be obtained. The features as well as effects of vertical seepage force, i.e., excess pore-pressure gradient in the vertical, were thoroughly studied. Finally, the influences of saturation degree, permeability coefficient and shear modulus of seabed soil are discussed in detail.

\section{Theoretical formulations and numerical model}

\subsection{Turbulent flow model}

The Reynolds-averaged continuity and momentum equations (RANS equations) in the Cartesian coordinate system are given by,

$$
\begin{aligned}
& \frac{\partial \rho_{w}}{\partial t}+\frac{\partial}{\partial x_{i}}\left(\rho_{w} U_{i}\right)=0 \\
& \rho_{w} \frac{D U_{i}}{D t}=-\frac{\partial p_{w}}{\partial x_{i}}+\frac{\partial}{\partial x_{j}}\left[\mu\left(\frac{\partial U_{i}}{\partial x_{j}}+\frac{\partial U_{j}}{\partial x_{i}}-\frac{2}{3} \delta_{i j} \frac{\partial U_{i}}{\partial x_{i}}\right)\right]+\frac{\partial}{\partial x_{j}}\left(-\rho_{w} \overline{u_{i}^{\prime} u_{j}^{\prime}}\right)
\end{aligned}
$$

where $\rho_{w}$ is the water density; $U_{i}$ is the $i$ th component of mean flow velocity; the operator $\frac{D}{D t}=\frac{\partial}{\partial t}+U_{i} \frac{\partial}{\partial x_{i}}$ in which $t$ is the time and $x_{i}$ is Cartesian coordinate; $p_{w}$ is the dynamic pressure; $\mu$ is the dynamic viscosity of water; $\delta_{i j}$ is the Kronecker delta; $u_{i}^{\prime}$ is the $i$ th component of fluctuating velocity, and the last term $\left(-\rho_{w} \overline{u_{i}^{\prime} u_{j}^{\prime}}\right)$ on the right hand of Eq. (2), called Reynolds stress tensor, can be obtained by,

$$
-\rho \overline{u_{i}^{\prime} u_{j}^{\prime}}=\mu_{t}\left(\frac{\partial U_{i}}{\partial x_{j}}+\frac{\partial U_{j}}{\partial x_{i}}\right)-\frac{2}{3}\left(\rho_{w} k+\mu_{t} \frac{\partial U_{i}}{\partial x_{i}}\right) \delta_{i j}
$$

in which $k$ is the turbulent kinetic energy and $\mu_{t}$ is the turbulent viscosity, which can be calculated by the SST turbulence model, as detailed in the following. 
Liang and Cheng (2005a) compared the performance of several turbulence models, including the standard $k-\varepsilon$, high and low Reynolds number $k-\omega$ and SGS (Smagorinsky's subgrid scale) models, for the simulation of the turbulent flow around a free spanning pipeline. It was found that the standard $k-\varepsilon$ model could predict the mean velocity quite well, and the $k$ - $\omega$ model with a no-slip boundary on the cylinder surface had better predictions of the vortex shedding. Combining the robustness of the $k-\varepsilon$ model with the superior behavior of $k$ - $\omega$ model in near-wall regions, the SST turbulence model proposed by Menter et al. (1994, 2003) was adopted in this study. As a supplement to RANS equations, the equations of SST model are formulated in terms $k$ (turbulent kinetic energy) and $\omega$ (turbulence specific dissipation rate),

$$
\begin{aligned}
& \left.\rho_{w} \frac{D k}{D t}=\widetilde{P}_{k}-\beta_{0}^{*} \rho_{w} k \omega+\frac{\partial}{\partial x_{i}}\left[\left(\mu+\sigma_{k} \mu_{t}\right)\right] \frac{\partial k}{\partial x_{i}}\right) \\
& \rho_{w} \frac{D \omega}{D t}=\frac{\rho_{w} \gamma}{\mu_{t}} \tilde{P}_{k}-\beta \rho_{w} \omega^{2}+\frac{\partial}{\partial x_{i}}\left[\left(\mu+\sigma_{\omega} \mu_{t}\right)\right] \frac{\partial \omega}{\partial x_{i}}+2\left(1-F_{1}\right) \rho_{w} \sigma_{\omega 2} \frac{1}{\omega} \frac{\partial k}{\partial x_{i}} \frac{\partial \omega}{\partial x_{i}}
\end{aligned}
$$

in which the turbulence production terms $\tilde{P}_{k}, P_{k}$ and the turbulent viscosity $\mu_{t}$ can be calculated as follows,

$$
\begin{aligned}
\tilde{P}_{k} & =\min \left(P_{k}, 10 \beta_{0}^{*} \rho_{w} k \omega\right) \\
P_{k} & =\mu_{t}\left(\frac{\partial U_{i}}{\partial x_{j}}\left(\frac{\partial U_{i}}{\partial x_{j}}+\frac{\partial U_{j}}{\partial x_{i}}\right)-\frac{2}{3}\left(\frac{\partial U_{i}}{\partial x_{i}}\right)^{2}\right)-\frac{2}{3} \rho_{w} k \frac{\partial U_{i}}{\partial x_{i}} \\
\mu_{t} & =\frac{\rho_{w} a_{1} k}{\max \left(a_{1} \omega, S F_{2}\right)}
\end{aligned}
$$

where $S=\sqrt{2 S_{i j} S_{i j}}$ is the magnitude of the strain-rate tensor. As a blend of the corresponding constants from the $k-\varepsilon$ and the $k-\omega$ model, the parameters $\beta, \gamma, \sigma_{k}$ and $\sigma_{\omega}$ can be calculated by $\beta=F_{1} \beta_{1}+\left(1-F_{1}\right) \beta_{2}, \gamma=F_{1} \gamma_{1}+\left(1-F_{1}\right) \gamma_{2}, \sigma_{k}=F_{1} \sigma_{k 1}+\left(1-F_{1}\right) \sigma_{k 2}, \sigma_{\omega}=F_{1} \sigma_{\omega 1}+\left(1-F_{1}\right) \sigma_{\omega 2}$,

respectively. The model constants $\beta_{1}=3 / 40, \gamma_{1}=5 / 9, \sigma_{k 1}=0.85, \sigma_{\omega 1}=0.5, \beta_{2}=0.0828, \gamma_{2}=0.44$, $\sigma_{k 2}=1.0, \sigma_{\omega 2}=0.856, a_{1}=0.31, \beta_{0}^{*}=0.09$. The two blending functions $F_{1}$ and $F_{2}$ are given by,

$$
\begin{aligned}
& F_{1}=\tanh \left\{\left\{\min \left[\max \left(\frac{\sqrt{k}}{\beta_{0}^{*} \omega l_{w}}, \frac{500 \mu}{\rho_{w} \omega l_{w}{ }^{2}}\right), \frac{4 \rho_{w} \sigma_{\omega 2} k}{C D_{\kappa \omega} l_{w}{ }^{2}}\right]\right\}^{4}\right\} \\
& F_{2}=\tanh \left[\left[\max \left(\frac{2 \sqrt{k}}{\beta_{0}^{*} \omega l_{w}}, \frac{500 \mu}{\rho_{w} \omega l_{w}{ }^{2}}\right)\right]^{2}\right]
\end{aligned}
$$


where $C D_{\kappa \omega}=\max \left(2 \rho_{w} \sigma_{\omega 2} \frac{1}{\omega} \frac{\partial k}{\partial x_{i}} \frac{\partial \omega}{\partial x_{i}}, 10^{-10}\right) ; l_{w}$ is the distance to the nearest wall. The blending function $F_{1}$ equals zero away from the surface ( $k$ - $\varepsilon$ model), and switches to one inside the boundary layer ( $k-\omega$ model).

\subsection{Seabed model}

It is known (Sumer and Fredsøe, 2006) that even moderate currents can induce alternate vortex formation and shedding around an isolated cylinder, at a rate determined by the flow velocity $\boldsymbol{U}$ and cylinder diameter $D$. As a result, the vortex street appears and fully develops behind the cylinder. For a free spanning pipeline, the formation of the vortex street is obviously affected by the underlying seabed (Bearman and Zdravkovich, 1978; Grass et al., 1984; Lei et al., 1999). At the same time, periodic changes in the flow velocity and pressure on the seabed have strong effects on the development of local scour around the pipeline (Sumer et al., 1988).

There are observable similarities between vortex streets and waves, especially when considering the variations of water pressure. Therefore, typical porous models for waveseabed interactions are used in this study to predict the seabed responses, including the excess pore-pressures, effective stresses and deformations of the soil skeleton. It has been recognized that there are two mechanisms controlling the wave-induced seepage flow (Zen and Yamazaki, 1990): the oscillatory and residual mechanisms. Thus, the overall excess porepressure $p$ can be expressed as,

$$
p=\tilde{p}_{\text {osc }}+\bar{p}_{r e s}
$$

where $\tilde{p}_{\text {osc }}$ is the oscillatory pore-pressure induced by the elastic deformation of the soil skeleton, while $\bar{p}_{\text {res }}$ represents the residual pore-pressure, which is period-averaged and defined by

$$
\bar{p}_{r e s}=\frac{1}{T} \int_{t}^{t+T} p d t
$$

where $T$ is the period of flow pressure oscillation. In essence, $\bar{P}_{r e s}$ is the result of accumulated plastic deformation of the soil skeleton under cyclic pressure actions.

\subsubsection{Oscillatory soil response}

In this paper, the sandy seabed is assumed to be homogeneous and isotropic, and the governing equations for oscillatory soil response in plane strain condition are given, 


$$
\begin{gathered}
G_{s} \nabla^{2} \tilde{u}_{s}+\frac{G_{s}}{1-2 v_{s}} \frac{\partial \tilde{\varepsilon}_{V}}{\partial x}=\frac{\partial \tilde{p}_{o s c}}{\partial x} \\
G_{s} \nabla^{2} \tilde{v}_{s}+\frac{G_{s}}{1-2 v_{s}} \frac{\partial \widetilde{\varepsilon}_{V}}{\partial z}=\frac{\partial \tilde{p}_{o s c}}{\partial z} \\
\nabla^{2} \tilde{p}_{o s c}-\frac{\gamma_{w} n_{s} \beta_{s}}{k_{s}} \frac{\partial \tilde{p}_{o s c}}{\partial t}=\frac{\gamma_{w}}{k_{s}} \frac{\partial \tilde{\varepsilon}_{V}}{\partial t}
\end{gathered}
$$

where $\tilde{u}_{s}$ and $\tilde{v}_{s}$ are the soil displacement in the $x$ and $z$ directions; $\widetilde{\varepsilon}_{V}=\partial \tilde{u}_{s} / \partial x+\partial \tilde{v}_{s} / \partial z$ represents the volume strain of the soil skeleton; $G_{s}$ is the soil shear modulus; $v_{s}$ is the Poisson's ratio; $k_{s}$ is the permeability coefficient; $n_{s}$ is the soil porosity; $\gamma_{w}$ is the unit weight of pore water; $\beta_{s}$ is compressibility of pore water and is defined by,

$$
\beta_{s}=\frac{1}{K_{w}}+\frac{1-S_{r}}{P_{w 0}}
$$

where $P_{w 0}$ represents the absolute pore-pressure; $K_{w}$ is the bulk modulus of water (equals $2 \times 10^{9} \mathrm{~N} / \mathrm{m}^{2}$, Yamamoto et al., 1978), and $S_{r}$ is the saturation degree of seabed.

\subsubsection{Residual soil response}

The one-dimensional (1D) equation for residual pore-pressure $\bar{P}_{r e s}$ was derived from the Biot's consolidation equation (Sumer and Fredsøe, 2002),

$$
c_{v 1} \frac{\partial \bar{p}_{r e s}}{\partial z}=\frac{\partial \bar{p}_{r e s}}{\partial t}+f_{r}
$$

in which $c_{v 1}$ is the coefficient for $1 \mathrm{D}$ consolidation and is calculated as,

$$
c_{v 1}=\frac{G_{s} k_{s}}{r_{w}} \frac{2\left(1-\mu_{s}\right)}{\left(1-2 \mu_{s}\right)}
$$

and the source term $f_{r}$ represents the total amount of pore-pressure generated per unit time and per unit volume of soil. Seed and Ranhman (1978) proposed a typical linear expression of $f_{r}$,

$$
f_{r}=\frac{\partial \bar{p}_{r e s}}{\partial t}=\frac{\sigma_{0}^{\prime}}{T}\left[\frac{\tau_{0}}{\alpha_{r} \sigma_{0}^{\prime}}\right]^{1 / \beta_{r}}
$$

where $\tau_{0}$ is the amplitude of shear stress in the soil; $\alpha_{r}$ and $\beta_{r}$ are functions of soil relative density $D_{r}=\left(r_{\max }-r_{s}\right) /\left(r_{\max }-r_{\min }\right)$, in which $r_{s}$ is the current void ratio, and $r_{\max }$ and $r_{\min }$ are the maximum and minimum void ratios, respectively. Based on test data from Alba et al. (1976), empirical expressions of $\alpha_{r}$ and $\beta_{r}$ are given by Sumer et al. (2012),

$$
\alpha_{r}=0.34 D_{r}+0.084
$$




$$
\beta_{r}=-0.37 D_{r}+0.46
$$

and $\sigma_{0}^{\prime}$ is the initial mean effective stress at a depth of $z$ below the seabed surface,

$$
\sigma_{0}^{\prime}=\frac{1+2 K_{0}}{3} \gamma_{s}^{\prime} z
$$

where $K_{0}$ is the coefficient of lateral earth pressure; $\gamma_{s}{ }^{\prime}=\gamma_{s}-\gamma_{w}$ is the effective unit weight of soil; $\gamma_{s}$ is the total unit weight of soil.

In this study, considering the nonuniformity of flow pressure along the seabed, a two dimensional (2D) governing equation for $\bar{P}_{\text {res }}$ (Jeng and Zhao, 2014; Zhao et al., 2014) is adopted and the corresponding source term $f_{r}$ is redefined as follows,

$$
\begin{aligned}
& c_{v 2} \nabla^{2} \bar{p}_{r e s}=\frac{\partial \bar{p}_{r e s}}{\partial t}+f_{r}(x, z) \\
& f_{r}(x, z)=\frac{\sigma_{0}^{\prime}}{T}\left[\frac{|\tilde{\tau}(x, z)|}{\alpha_{r} \sigma_{0}^{\prime}}\right]^{1 / \beta_{r}}
\end{aligned}
$$

in which $\tilde{\tau}(x, z)$ is the amplitude of shear stress at a location $(x, z) ; c_{v 2}$ is the coefficient for the $2 \mathrm{D}$ plane strain consolidation and is calculated as,

$$
c_{v 2}=\frac{G_{s} k_{s}}{r_{w}} \frac{1}{\left(1-2 \mu_{s}\right)}
$$

\subsection{Computational domains and boundary conditions}

As shown in Fig. 1, the numerical model contains two computational domains: domain 1 is to simulate the flow field around the suspended pipeline, and domain 2 is the porous seabed model proposed above. The whole computational domain covers the range $x / D=-10$ to $x / D=$ 20 in the horizontal direction, and $z / D=-4$ to $z / D=4$ in the vertical direction. The incoming boundary layer flow is along the positive direction of the $x$ axis and has a free-stream velocity of $U_{0}=U_{x}(z=4 D)$, where $U_{x}(z)$ is the profile of horizontal velocity. The distance between the lowest point of the pipeline and seabed surface is $h$, and the current gap ratio is $h / D$.

\subsubsection{Boundary conditions for domain 1 (flow field)}

(1) boundary $\mathrm{AB}$ and the pipeline surface: at these two boundaries, a no-slip condition is imposed whereby the horizontal and vertical velocities are set to zero. The pressure is obtained by applying the momentum equations in the direction normal to these boundaries. The turbulent kinetic energy $k$ equals zero, and the turbulence specific dissipation rate $\omega$ is 
obtained by $\omega=10 \frac{6 v}{\beta_{1}(\Delta z)^{2}}$, where $v$ is the kinematic viscosity of water and $\Delta z$ is the distance to the first grid point above the surface. The first near-wall grid size $z^{+}$should be small enough, e.g. $z^{+}=\Delta z \cdot u * / v<1$, where $u *$ is the friction velocity;

(2) boundary BC: this is the outflow boundary of the flow field. Zero normal gradient of velocity and turbulence quantities are implemented. Pressure is given a reference value of $p_{\mathrm{w}}(\mathrm{z})=0$ at the outflow boundary.

(3) boundary CD: it is a frictionless slip wall i. e. with vertical velocities set to zero, and horizontal velocities and scalar hydrodynamic quantities having zero gradients.

(4) boundary $\mathrm{AD}$ : a fully developed turbulent velocity profile $\left(U_{x}(z), U_{z}(z)\right)$ is specified as

$$
\begin{aligned}
& \frac{U_{x}(z)}{u^{*}}=\frac{z u^{*}}{v}, \frac{z u^{*}}{v} \leq 11.63 \\
& \frac{U_{x}(z)}{u^{*}}=\frac{1}{\kappa} \ln \left(9.0 \frac{z u^{*}}{v}\right), \frac{z u^{*}}{v} \geq 11.63 \\
& U_{z}(z)=0
\end{aligned}
$$

where $\kappa=0.42$ is the von Kármán constant. The pressure is obtained by applying the momentum equations in the direction normal to boundary AD. The profiles of dimensionless turbulent kinetic energy $k(z)$, turbulence length scale $l(z)$ and specific dissipation rate $\omega(z)$ are,

$$
\begin{aligned}
& k(z)=\max \left\{C_{\mu}^{-1 / 2}\left(1-\frac{z}{\delta}\right)^{2} u^{*^{2}}, 0.0005 U_{0}^{2}\right\} \\
& l(z)=\min \left\{\frac{\kappa z}{1+1.5 z / \delta}, C_{\mu} \delta\right\} \\
& \omega(z)=\frac{k(z)^{1 / 2}}{\beta_{0}^{* 1 / 4} l(z)}
\end{aligned}
$$

in which $C_{\mu}=0.09$ and $\delta=4 D$ is the vertical dimension of flow domain 1 .

\subsubsection{Boundary conditions for domain 2 (porous seabed)}

(1) boundary AB: $\tilde{p}_{o s c}=p_{b}, \bar{p}_{\text {res }}=0$, where $p_{b}$ is the instant pressure on the seabed;

(2) boundaries AF and BE: $\partial \tilde{p}_{o s c} / \partial x=\partial \bar{p}_{r e s} / \partial x=0, \tilde{u}_{s}=0$;

(3) boundary EF: $\partial \tilde{p}_{\text {osc }} / \partial z=\partial \bar{p}_{r e s} / \partial z=0, \tilde{u}_{s}=\tilde{w}_{s}=0$.

\subsection{One-way coupling of the flow and seabed models}

In the simulation, three segregated solve steps are used to couple the turbulent flow model 
and porous seabed model: in the first step, the flow field around a free spanning pipeline is numerically simulated; second, the water pressure on the seabed $p_{b}$ during the whole simulation time is extracted and stored; in the final step, the water pressure $p_{b}$ varying with time is imposed on the seabed surface, and then the seabed model is solved to obtain the seabed response, including the excess pore-pressures, effective stresses and deformations of the soil skeleton.

\section{Numerical results and interpretations}

\subsection{Flow field around the pipeline}

The SST turbulence model is adopted in this study to simulate the complicated flow field around the free spanning pipeline. To validate the reliability of the SST model, the calculated flow velocities are compared with previous experimental results from Jensen (1987). In Jensen's experiments, the Reynolds number based on a free-stream velocity $U_{0}$ and a cylinder diameter $D$ is equal to 7000, and the current gap ratio is $h / D=0.37$. As shown in Figs. 2, 3, 4 and 5, at six cross-sections downstream of the pipeline $x=1.0 D, 1.5 D, 2.5 D, 4.5 D, 8.5 D$ and $16.5 \mathrm{D}$, both the mean and fluctuating flow velocities in the horizontal and vertical directions are presented for comparisons. It is shown that the mean horizontal $\left(U_{x}\right)$ and vertical $\left(U_{z}\right)$ velocities predicted by the SST model coincide roughly with the measured data. Only a few differences appear in the sections near the pipeline $(x=1.0 D, 1.5 D, 2.5 D)$. In this paper, the velocity fluctuation is defined as the RMS of the deviation of velocity samples to the longtime-averaged value,

$$
\begin{aligned}
& \Delta U_{x}=\sqrt{\frac{\sum_{i=1}^{N}\left(U_{x i}-\bar{U}_{x}\right)^{2}}{N}} \\
& \Delta U_{z}=\sqrt{\frac{\sum_{i=1}^{N}\left(U_{z i}-\bar{U}_{z}\right)^{2}}{N}}
\end{aligned}
$$

where $N$ is the total number of velocity samples; $U_{x i}$ and $U_{z i}$ are the velocity samples; $\bar{U}_{x}$ and $\bar{U}_{z}$ are the averaged velocities over a long period of time in $x$ and $z$ directions, respectively. It is found that the fluctuation velocity profiles predicted by SST model coincide well with the measurements at most of the cross-sections $(x=1.0 D, 1.5 D, 2.5 D$ and $4.5 D)$, especially in the region below the suspended pipe, although the fluctuation is obviously under-predicted in the region exceeding $8.5 \mathrm{D}$ from the pipeline (this region is not the focus of 
this paper). It is thought that the main characteristics of flow velocity near the seabed have been captured by this numerical simulation.

It is known that vortices are shed alternatively from the top and bottom of the pipeline and they induce dynamic loads on the pipeline, which could lead to Vortex Induced Vibration (VIV) of pipeline (Sumer and Fredsøe, 2006). The frequency of vortex shedding $(f)$ is consistent with that of VIV forces. In this study, the drag and lift coefficients $\left(C_{P D}\right.$ and $\left.C_{P L}\right)$ for the VIV forces are defined as,

$$
C_{P D}=\frac{F_{P D}}{0.5 \rho_{w} D U_{0}^{2}}, \quad C_{P L}=\frac{F_{P L}}{0.5 \rho_{w} D U_{0}^{2}}
$$

where $F_{P D}$ and $F_{P L}$ are the longitudinal and transverse forces acting on the pipeline. Fig. 6 shows the variations of two coefficients with the dimensionless period $\left(t U_{0} / D\right)$. The numerical results coincide with previous works (Sumer et al., 1991; Sumer and Fredsøe, 2006; Fuhrman et al., 2014) that the lift coefficient is not symmetrical about the axis of $C_{P L}=0$. The maximum $C_{P L}$ is about 1.2 and the minimum $C_{P L}$ equals -1.0. The dimensionless period of VIV forces is approximately 5 . This suggests the Strouhal number $S t=f D / U_{0} \approx 0.2$, which is supported by Lei et al. (1999). Thus, in this case, the frequency of vortex shedding around the pipeline is about 0.016 .

As plotted by the velocity vectors in Fig. 7 , in a typical period $\left(t_{0} \rightarrow t_{0}+3 T / 4\right)$, the vortices shed from the pipeline would interact with the underlying seabed, inducing obvious velocity fluctuations near the seabed. Previous studies (Sumer et al., 1988; Li and Cheng, 2001) indicated that the fluctuating horizontal velocity had a significant effect on the local scour around the pipelines. In these works, the seabed was assumed to be impermeable. However, for the case of permeable seabed, the mass and momentum transfer takes place across the interface between the water and seabed, and the seepage flow appears inside the seabed. Thus, it may be inappropriate to ignore the vertical parameters, such as the vertical flow velocity or pressure, in the analysis of the local scour around the pipeline. In the work of Francalanci $e t$ al. (2008), the nonhydrostatic pressure caused by vertical seepage flow was introduced to consider the influence of the vertical parameter on the sediment incipient motion.

Similar to the horizontal flow velocity, the water pressure acting on the seabed can be divided into the mean and fluctuating components. In Fig. 8, the pressures measured by Bearman and Zdravkovich (1978) are plotted to validate the pressures calculated by the SST model. For comparison, a gap ratio $h / D=0.4$ and a Reynolds number of 15000 are adopted in the simulation. The mean and fluctuating pressure coefficients $C_{p}$ are calculated as follows, 


$$
C_{p}=\frac{p_{b}-p_{0}}{0.5 \rho_{w} U_{0}^{2}}
$$

in which $p_{0}$ is the reference pressure. It is shown that the mean $C_{p}$ roughly agrees with the measured $C_{p}$, and the range of fluctuating $C_{p}$ nearly covers all measured data, especially in the downstream zone of the pipeline.

Fig. 8 also shows two features of water pressure acting on the seabed: the non-uniform along the seabed and the fluctuation with time. It is shown that the negative water pressure only appears in the upstream zone of the pipeline, while the positive pressure mainly acts on the seabed surface near the pipeline and reaches its peak just under the pipeline. In the downstream zone, the positive pressure gradually changes to zero with an increasing distance away from the pipeline. Another feature is the pressure fluctuation with time due to the periodic vortex shedding behind the pipeline. As shown in Fig. 8, the pressure fluctuations are significant in the zone near the pipeline, and become much smaller away from the pipeline (approximately $8 D$ ). This feature of pressure fluctuation is similar to that of wave-induced water pressure, under which the deformation of seabed soil easily occurs, excess porepressure develops, and thus the stability of bed particles may be largely changed. Therefore, to study the sediment incipient motion around the pipeline, both the non-uniform and fluctuation features of water pressure should be considered in the simulation.

\subsection{Vortex shedding-induced seabed response}

In this section, the dynamic response of seabed soil will be studied first through a specific case. It is known that for near-bed flow past a submarine pipeline in the field, the Reynolds number $R e$ generally lies in the subcritical flow regime $\left(150<R e<3.0 \times 10^{5}\right)$. Therefore, a pipeline diameter $D=0.3 \mathrm{~m}$ and a free-stream velocity $U_{0}=0.6 \mathrm{~m} / \mathrm{s}$ are used in this case. Previous experimental results (Grass et al., 1984; Lei et al., 1999) revealed that vortex shedding in a subcritical flow regime is strong and periodic, but is completely suppressed when $h / D$ is less than approximately 0.3 . Thus, $h / D$ is set to 0.4 and all other parameters are given in Table. 1. By taking the water pressures calculated by turbulent flow model as the boundary conditions for the seabed model in section 2.2, the excess pore-pressures (both oscillatory and residual pore-pressures) within the seabed can be solved. The accuracy and reliability of the seabed model have been verified through comparing with experimental data (Tsai and Lee, 1995; Pan et al., 2007; Sumer et al., 2012; Liu and Jeng, 2013; Kirca et al., 2013) and analytical solutions (Hsu and Jeng, 1994) in the works of Jeng and Zhao (2014), Zhao et al. (2014). 


\subsubsection{Oscillatory pore-pressure}

The oscillatory pore-pressure $\tilde{P}_{\text {osc }}$ is directly caused by the elastic volume deformation of the soil skeleton, so $\tilde{P}_{o s c}$ in the seabed has a feature of cyclic oscillation under the effect of water pressure induced by periodic vortex shedding. The variations of oscillatory porepressure in one period $\left(t_{0} \rightarrow t_{0}+5 T / 6\right)$ are shown in Fig. 9(a), (b), (c), (d), (e) and (f). It is found that the maximum $\tilde{P}_{\text {osc }}$ always appears on the seabed surface, and moves circularly from the pipeline bottom to the downstream zone under periodic pressure conditions.

\subsubsection{Residual pore-pressure}

Under the effect of the fluctuating flow pressure induced by vortex shedding, the residual mechanism works and produces the build-up of residual pore-pressure in a sandy seabed. Residual pore-pressure $\bar{P}_{r e s}$ is the result of accumulated plastic deformation of the soil skeleton. Under the conditions of cyclic water pressure, $\bar{P}_{\text {res }}$ builds up in the seabed and dissipates at the same time, until reaching an equilibrium state. Fig. 10 shows the accumulation process of the residual pore-pressure $\bar{P}_{\text {res }}$ with time $(t=50,100,150,250,500$, 900 s). It is found that the maximum $\bar{P}_{r e s}$ appears at a certain location that is below the seabed $(z \approx-0.04 \mathrm{~m})$ and downstream of the pipeline $(\mathrm{x} \approx 0.42 \mathrm{~m})$. This is quite different from that of the oscillatory pore-pressure $\tilde{P}_{o s c}$.

\subsubsection{Physics on the incipient motion of a bed particle}

For the sandy seabed, there is no cohesive force among sediment particles. Therefore, a cohesionless sediment particle resting on a porous bed is generally subjected to its submerged weight force $W$, drag force $F_{D}$, lift force $F_{\mathrm{L}}$ and vertical seepage force $F_{S}$ (for simplicity only the vertical component of seepage force is considered), as shown in Fig. 11. All bed particles are assumed to be spheres with a uniform diameter of $d$, and the forces are calculated as,

$$
\begin{aligned}
& W=\left(\gamma_{d}-\gamma_{w}\right) \frac{\pi d^{3}}{6} \\
& F_{D}=C_{D} \frac{\pi d^{2}}{4} \frac{\rho_{w} u_{b}^{2}}{2} \\
& F_{L}=C_{L} \frac{\pi d^{2}}{4} \frac{\rho_{w} u_{b}^{2}}{2}
\end{aligned}
$$

where $\gamma_{d}$ is the unit weight of a sediment particle; $C_{D}$ and $C_{L}$ are the drag and lift coefficients respectively; $u_{\mathrm{b}}$ is the approach flow velocity on the particle at the bed surface. The seepage 
force $F_{S}$ is caused by the vertical variation of nonhydrostatic pressure $p$ at the seabed surface and is expressed as,

$$
F_{S}=\frac{\partial p}{\partial z} \frac{\pi d^{3}}{6}
$$

The driving force for the motion of a bed particle is denoted as $F_{D}$, and the resistance force $F_{R}=f_{R} \times\left(W-F_{S}-F_{L}\right)$, where $f_{R}$ is the Coulomb friction coefficient. In most previous works, the contribution of seepage force $\left(F_{S}=0\right)$ was ignored, and thus the dimensionless Shields number $\theta$ was defined as,

$$
\theta=\frac{\tau_{b}}{\left(\gamma_{d}-\gamma_{w}\right) d}
$$

where $\tau_{b}$ is the bed shear stress. Eq. (40) is a simplified formula due to the absence of a seepage force $F_{S}$. However, the seepage flow through a porous boundary $\left(F_{S} \neq 0\right)$ may cause a remarkable modification to the submerged weight of bed particle. Here, a scaling factor $e$ is introduced to define the ratio of seepage force to the submerged particle weight,

$$
e=\frac{F_{S}}{W}=\frac{\partial p / \partial z}{\gamma_{d}-\gamma_{w}}
$$

and then, we could have the "real" submerged weight force $W^{\prime}$ as follows,

$$
W^{\prime}=W-F_{S}=(1-e) W=(1-e)\left(\gamma_{d}-\gamma_{w}\right) \frac{\pi d^{3}}{6}
$$

Thus, the original Shields number is modified by including the seepage contribution,

$$
\theta^{*}=\frac{\tau_{b}}{(1-e)\left(\gamma_{d}-\gamma_{w}\right) d}=\frac{1}{1-e} \theta
$$

in which the scaling factor $e$ should be less than 1. It is found in Eq. (41) that if the factor $e<$ 0 , representing the downward seepage flow, $\theta^{*} / \theta$ becomes less than 1 and will gradually reduce with an decreasing $e$; while the upward seepage occurs with $0<e<1, \theta^{*} / \theta$ exceeds 1.0 and shows a sharp increase trend with an increasing $e$. For the factor $e=0.50, \theta^{*} / \theta$ increases up to 2.0 that means the Shields number increases two times if considering the contribution of the seepage force. Therefore, in some cases, the seepage effect on the particle incipient motion may be significant and should not be ignored in the analysis.

\subsubsection{Contributions of seepage flow to sediment incipient motion}

Fig. 12 shows the distributions of time-averaged, maximum and minimum shear stresses along the seabed surface. It has been acknowledged that the maximum bed shear stress plays a more important role in the development of local scour (Li and Cheng, 2001; Sumer and 
Fredsøe, 2002). Thus, the maximum shear stress is substituted into Eq. (40) to calculate the Shields number, and the distribution of original Shields number (no seepage effect) is plotted as a blue solid line in Fig. 13. In this figure, the critical Shields number for the incipient motion of sediment particles on a flat seabed is also given by (Soulsby, 1997),

$$
\theta_{c r}=0.30 /\left(1+1.2 D^{*}\right)+0.055\left[1-e^{-0.02 D^{*}}\right]
$$

where the dimensionless diameter of sediment particle $D^{*}=\left[g\left(\gamma_{d} / \gamma_{w}-1\right) / v^{2}\right]^{1 / 3} d$ in which $v$ is the kinematic viscosity of water and $g$ is the gravitational acceleration. It is found that in the region beneath the suspended pipeline $(-1.0 D<\mathrm{x}<2.0 D)$, the critical Shields number is exceeded so that the local scour is expected to occur in this region. However, it should be noted that in the present model, the depicted seabed conditions only show the early times of the flow, before the bed is scoured.

As discussed in section 2.2, there are two mechanisms controlling the seepage flow: the oscillatory and residual mechanisms. Correspondingly, two seepage forces, which can be referred to "oscillatory" and "residual" seepage forces, appear and act on the bed particle. Here, two scaling factors $\tilde{e}$ (oscillatory factor) and $\bar{e}$ (residual factor) are used to define the contributions of oscillatory and residual seepage forces to the sediment incipient motion,

$$
\begin{gathered}
\tilde{e}=\frac{\partial \tilde{p}_{o s c} / \partial z}{\gamma_{d}-\gamma_{w}} \\
\bar{e}=\frac{\partial \bar{p}_{r e s} / \partial z}{\gamma_{d}-\gamma_{w}}
\end{gathered}
$$

As plotted in Fig. 14(a), the oscillatory factor $\tilde{e}$ fluctuates with periodic vortex shedding. It can be seen that obvious negative $\tilde{e}$ exists in the region of $-1.0 D<\mathrm{x}<3.0 D$ below the pipe, and the minimum of $\tilde{e}$ is about -0.14 at the location of $1.5 \mathrm{D}$ downstream of the pipeline. According to Eq. (43), the Shields number $\theta$ at $x=1.5 D$ is reduced by $13 \%$ in this location. The positive $\tilde{e}$ also exists in Fig. 14(a) and shows two large peaks in which the bigger one $\tilde{e}$ $=0.18(22 \%$ increase in $\theta)$ lies just below the pipeline, and the smaller one $\tilde{e}=0.15(18 \%$ increase in $\theta$ ) exists near $x=1.5 D$ downstream of the pipeline. Fig. 14 (b) gives the development of the residual factor $\bar{e}$ with time $t=50,100,150,250,500,900 \mathrm{~s}$. Finally, the residual factor $\bar{e}$ reaches a stable parabolic-like profile with a single peak of $\bar{e}=0.11$, which would increase the Shields number $\theta$ by $12 \%$. Larger $\bar{e}$ mainly lies in the region from $x=$ $1.0 D$ to $2.0 D$.

As shown in Fig. 14, the oscillatory seepage contributes to affect the sediment incipient 
motion in a wider region of the seabed than the residual seepage does. The oscillatory seepage force works immediately once the vortices shed periodically from a suspended pipeline, so the original Shields number can be modified by $\theta /(1-\tilde{e})$ in the early stage and the corresponding curve is shown in Fig. 13. It is noted that the Shields number in the region beneath the pipeline ( $x=-1.0 D$ to $2.0 D$ ) is largely increased, and the criterion $\theta /(1-\tilde{e})>\theta_{c r}$ is satisfied in a little wider region of the seabed. If including the contribution of residual seepage with time, the final curve for modified Shields number $\theta /(1-(\tilde{e}+\bar{e}))$ is marked by a red dash-dot line in Fig.13. It is shown that the residual seepage mainly increases the Shields number in the zone from $x=1.0 D$ to $2.0 D$. In addition, it should be noticed that Diplas et al. (2008) and Valyrakis et al. (2010) suggested that for the oscillatory force, except for the magnitude of the force, its duration is also important in determining the threshold for sediment incipient motion. The results of oscillatory seepage force in this paper only reflect the effect of the force magnitude, and thus may not be accurate enough in some cases, such as for a quick vertex shedding.

\subsection{Parametric analysis}

The soil response under the influence of a periodically shedding vortex actually depends on many parameters of the porous seabed, such as the saturation degree $S_{r}$, permeability coefficient $k_{s}$ and shear modulus of soil $G_{s}$. In this section, quantitative comparisons will be made to assess the different effects of these parameters. To make the analysis clear, the so called "envelope curve" for the oscillatory factor $\tilde{e}$ along the seabed surface is proposed to represent the maximum $\tilde{e}$ experienced in one period of vortex shedding. The distribution of the final stable residual factor $\bar{e}$ is presented for discussion as well.

\subsubsection{Effect of saturation degree $S_{r}$}

It is believed that most marine sediments have a high degree of saturation that is close to 1.0, but the existence of full saturation $\left(S_{r}=1.0\right)$ is rare. For comparison, saturation degrees $S_{r}$ $=1.0,0.98,0.96,0.94,0.92$ and 0.90 were adopted in numerical calculations, and corresponding envelop curves for the oscillatory factor $\tilde{e}$ are plotted in Fig. 15. It is shown that with a decrease of saturation degree $S_{r}$, the maximum $\tilde{e}$ on the seabed shows an obvious increase, especially in the region near the two peaks of maximum $\tilde{e}$ that are at about $x=0 D$ and 1.5D. At the location of $x=0 D$, the maximum $\tilde{e}$ increases from $0.15(18 \%$ increase in $\theta)$ to 0.35 (54\% increase in $\theta$ ); for the location $x=1.5 D$, the maximum $\tilde{e}$ changes from 0.14 ( $16 \%$ increase in $\theta)$ to 0.31 (45\% increase in $\theta$ ). 
Fig. 16 gives the effect of the degree of seabed saturation $S_{r}$ on the final residual factor $\bar{e}$. It is shown that if the saturation degree $S_{r}$ decreases gradually from 1.0 to 0.90 , the peak of the residual factor $\bar{e}$ only has a slight increase from 0.105 (12\% increase in $\theta$ ) to 0.153 ( $18 \%$ increase in $\theta$ ). It seems that the saturation degree of the seabed could significantly affect the oscillatory factor $\tilde{e}$ along the seabed surface but has less impact on the residual factor $\bar{e}$.

\subsubsection{Effect of the permeability coefficient $k_{s}$}

The permeability of the porous seabed is also an important factor that impacts the development of excess pore-pressure inside the seabed. Here, different permeability coefficients $\left(k_{s}=10^{-4}, 10^{-5}, 5 \times 10^{-6}, 10^{-6}, 5 \times 10^{-7}, 10^{-7} \mathrm{~m} / \mathrm{s}\right)$ were used in the simulation, and their effects on the envelope curve of oscillatory factor $\tilde{e}$ and final residual factor $\bar{e}$ are plotted in Figs. 17 and 18, respectively. Similar to the effect of saturation degree $S_{r}$, the decrease of seabed permeability leads to a remarkable increase of the maximum $\tilde{e}$. At the peak close to $x=0 D$, the maximum $\tilde{e}$ increases from 0.12 (14\% increase in $\theta)$ to $0.30(43 \%$ increase in $\theta$ ); for another peak near $x=1.5 D$, the maximum $\tilde{e}$ grows from $0.15(18 \%$ increase in $\theta$ ) to 0.21 (27\% increase in $\theta$ ).

It is clear that the maximum oscillatory factor $\tilde{e}$ is affected by the seabed permeability, but it is found that it has a greater effect on the residual factor $\bar{e}$, as shown in Fig. 18. When the permeability coefficient $k_{s}$ decreases from $10^{-4} \mathrm{~m} / \mathrm{s}$ to $5 \times 10^{-6} \mathrm{~m} / \mathrm{s}$, the residual factor $\bar{e}$ only increases slightly; but when the permeability coefficient $k_{s}$ is further reduced to be lower than $10^{-6} \mathrm{~m} / \mathrm{s}$, the residual factor $\bar{e}$ shows a significant increase. In particular, when $k_{s}=10^{-7} \mathrm{~m} / \mathrm{s}$, the residual factor $\bar{e}$ at $x=1.5 \mathrm{D}$ increases up to 0.67 , in which case the modified shields number becomes three times of original shields number due to high pore-pressure variation in the vertical.

\subsubsection{Effect of the soil shear modulus $G_{s}$}

The shear modulus $G_{s}$ of soil could vary from approximately $10^{8} \mathrm{~N} / \mathrm{m}^{2}$ for dense sand or gravel to $10^{6} \mathrm{~N} / \mathrm{m}^{2}$ for loose sand or silt, so numerical investigations into the cases of $G_{s}=10^{6}$, $5 \times 10^{6}, 10^{7}, 5 \times 10^{7}, 10^{8} \mathrm{~N} / \mathrm{m}^{2}$ were performed. It is found in Fig. 19, with the increase of the soil shear modulus, the curve of maximum oscillatory factor $\tilde{e}$ only changes slightly. At $x=$ $0 D$, the maximum $\tilde{e}$ increases from 0.18 (22\% increase in $\theta$ ) to $0.21(27 \%$ increase in $\theta$ ); at $x=1.5 D$, the maximum $\tilde{e}$ changes from approximately 0.13 (15\% increase in $\theta$ ) to 0.17 (20\% increase in $\theta)$.

However, as shown in Fig. 20, the final residual factor $\bar{e}$ shows a relatively large 
increasing trend with the decrease of the soil shear modulus $G_{s}$. This effect of the soil shear modulus $G_{s}$ is opposite to its effect on the maximum oscillatory factor $\tilde{e}$ that is illustrated in Fig. 16. When the shear modulus $G_{s}$ decreases from $10^{8} \mathrm{~N} / \mathrm{m}^{2}$ to $10^{6} \mathrm{~N} / \mathrm{m}^{2}$, the residual factor $\bar{e}$ could increase from 0.01 (only $1 \%$ increase in $\theta$ ) up to approximately 0.37 (up to $59 \%$ increase in $\theta$ ).

\section{Discussions}

As described in this paper, the vortices that shed periodically from a free spanning pipeline induce continuous oscillations of water pressure on the seabed. As a consequence, two types of excess pore pressures, oscillatory and residual pore-pressures, develop inside the seabed with time. For a cohesionless sediment particle resting on the seabed, its submerged weight will be reduced due to the vertical upward seepage force at the seabed surface. This kind of seepage force is caused by the variations of the oscillatory and residual pore-pressures in the vertical. Through detailed numerical studies, it is found that the oscillatory seepage force acts on a relatively wider zone of the seabed surface than the residual seepage force does. Both the saturation degree and permeability of the seabed obviously affect the distribution and magnitude of the oscillatory seepage force acting on the bed particle, but the soil shear modulus only has a small impact. The seabed parameters that have the strong impact on the residual seepage force are the seabed permeability and the soil shear modulus, and the effect of saturation degree is smaller. It is important to note that the above studies are based on an ideal assumption that these three parameters, the saturation degree, permeability coefficient and soil shear modulus, are independent of each other. In fact, this assumption is oversimplified. For a sandy seabed, the large shear modulus always appears with a low soil porosity and permeability coefficient, and its saturation degree in the field depends on many factors. Therefore, the incipient motion of bed particles around the suspended pipeline should be investigated on a case-by-case basis.

This paper is an attempt to include the seepage force in the numerical analysis of sediment incipient motion around the suspended pipeline. Based on both the porous media theory and the soil mechanics, the permeable and deformable characteristics of the sandy seabed are considered in the simulation. To simplify the question, the effect of seepage flow on the nearseabed fluid flow was ignored. Previous experimental studies have noted that the near-seabed flow velocity may be reduced due to vertical upward seepage, but test results are taken from qualitative analysis or phenomenal descriptions, and the physics behind it still remains 
unclear. Therefore, only the impact of seepage on the resistance force of a bed particle is investigated here. In addition, the sediment particle is assumed to be alone on the porous seabed in this paper (Fig. 21(b)). However, the real situations in the field are quite complicated. As shown in Fig. 21, Liu and Chiew (2012) have presented four different positions of sediment particles resting on a bed surface, and pointed out that Positions 2 to 4 did not represent a general case in the field. For the conditions of Position 1 and 3, the impact of the bed shear stress on particle stability is considerably less significant because of shielding effects. Consequently, the vertical seepage force plays a more critical role in raising such particles away from the underlying seabed.

\section{Conclusions}

This paper proposed an integrated numerical model, coupling the SST turbulence model with a $2 \mathrm{D}$ porous seabed model, to study the effect of seepage flow on the sediment incipient motion around a free spanning pipeline. It was shown that the SST model could predict the complicated flow field around the suspended pipeline quite well. By taking the calculated water pressure as the boundary conditions of seabed model, two kinds of excess porepressures in the seabed, which are the oscillatory and residual pressures, were investigated in detail. Hence, the traditional dimensionless Shields number was modified to include the contributions of seepage forces that are caused by vertical variations of oscillatory and residual pressures. Through a series of numerical studies, it is concluded when considering the permeability and deformability of the seabed, the mobility of bed particles around the suspended pipeline may be largely enhanced in some cases. Detailed conclusions can be drawn as follows:

(1) The oscillatory seepage force along the seabed surface generally has two obvious peaks (below and downstream of the pipeline), and shows a wider region of impact than the residual seepage force, which only increases in some specific locations downstream of the pipeline.

(2) For the decreases in both the saturation degree and the permeability of the seabed, a significant increase in the oscillatory seepage force is possible and thus the mobility of bed particles could be enhanced; conversely, a decrease in the soil shear modulus would lead to a slightly reduced oscillatory seepage force exerted on the particles.

(3) With a gradual decrease in the saturation degree from 1.0 to 0.9 , the residual seepage force only increases slightly; when the seabed permeability decreases, the residual seepage force increases dramatically and the submerged particle weight is largely reduced; with a smaller soil shear modulus, the residual seepage force shows a big increasing trend that is 
opposite to its effect on the oscillatory seepage force.

(4) In conclusion, for a permeable and deformable seabed, a smaller saturation degree and seabed permeability increases both the oscillatory and residual seepage forces acting on the bed particles, and thus obviously enhance the particle mobility; however, for a seabed with a smaller soil shear modulus, the magnitude of oscillatory seepage force is reduced, and the effect of residual seepage force becomes dominated and cannot be ignored in the determination of the sediment incipient motion.

\section{Acknowledgments}

The authors would like to acknowledge the support of the Grant No. 51779220,51209183 and 51325901 from the National Natural Science Foundation of China, Zhejiang Provincial Natural Science Foundation of China (LY15E090002), Key Research and Development program of Zhejiang Province (2018C03031).

\section{References}

Alba, P. D., Seed, H. B., Chan, C. K., 1976. Sand liquefaction in large-scale simple shear tests. J. Geotech. Eng. Div., ASCE, 102(GT9), 909-927.

Bearman, P. W., Zdravkovich, M. M., 1978. Flow around a circular cylinder near a plane boundary. J. Fluid Mech., 89(1), 33-47.

Cheng, N. S., 1997. Seepage effect on open-channel flow and incipient sediment motion. Ph.D. thesis, Nanyang Tech. Univ., Singapore.

Chiew, Y. M., Parker, G., 1994. Incipient sediment motion on non-horizontal slopes. J. Hydraul. Res., 32(5), 649-660.

Diplas, P., Dancey, C.L., Celik, A.O., Valyrakis, M., Greer, K., Akar, T., 2008. The role of impulse on the initiation of particle moveunder turbulent flow conditions. Science, 322(5902), 717-720.

Hsu, J. R. C., Jeng, D.-S., 1994. Wave-induced soil response in an unsaturated anisotropic seabed of finite thickness. Int. J. Numer. Anal. Methods Geomech., 18(11), 785-807.

Francalanci, S., Parker, G., Solar, L., 2008. Effect of seepage-induced non-hydrostatic pressure distribution on bed-load transport and bed morphodynamics. J. Hydraul. Eng., ASCE, 134(4), 378-389.

Fuhrman, D. R., Baykal, C., Sumer, B. M., Jacobsen, N. G., Fredsøe, J., 2014. Numerical simulation of wave induced scour and backfilling processes beneath submarine pipelines. Coastal Eng., 94, 10-22. 
Grass, A. J., Raven, P. W. J., Stuart, R. J., Bray, J. A., 1984. The influence of boundary layer velocity gradients and bed proximity on vortex shedding from free spanning pipelines. $\mathrm{J}$. Energy Res. Tech., 106, 70-78.

Jeng, D.-S., Zhao, H. Y., 2014. Two-dimensional model for accumulation of pore-pressure in marine sediments. J. Waterw. Port Coast. Ocean Eng., ASCE, 04014042, 1-12.

Jensen, B. L., 1987. Large-scale vortices in the wake of a cylinder placed near a wall. Proc. 2nd Int. Conf. on Laser-Anemometry-Advances and Applications, Strathclyde, UK, 153163.

Kirca, V. S. O., Sumer, B. M., Fredsøe, J., 2013. Residual liquefaction of seabed under standing waves. J. Waterway, Port, Coastal, Ocean Eng., 139(6), 489-501.

Lei, C., Cheng, L., Kavanagh, K., 1999. Reexamination of the effect of a plane boundary on force and vortex shedding of a circular cylinder. J. Wind Eng. Ind. Aerodyn., 80(3), 263286.

Li, F., Cheng, L., 2001. Prediction of lee-wake scouring of pipelines in currents. J.Waterw. Port Coast. Ocean Eng., 127 (2), 106-112.

Liang, D. F., Cheng, L., 2005a. Numerical modeling of flow and scour below a pipeline in currents Part I. Flow simulation. Coast. Eng., 52, 25-42.

Liang, D. F., Cheng, L., Li, F. J., 2005b. Numerical modeling of flow and scour below a pipeline in currents Part II. Scour simulation. Coast. Eng., 52, 43-62.

Liu, X. X., Chiew, Y. M., 2012. Effect of seepage on initiation of cohesionless sediment transport. Acta Geoohys., 60(6), 1778-1796.

Liu, B., Jeng, D.-S., 2013. Laboratory study for pore pressure in sandy bed under wave loading. Proc., of the 23rd Int. Offshore and Polar Engineering Conf., ISOPE, Cupertino, CA, 1432-1437.

Madsen, O. S., Grant, W. D., 1976. Quantitative description of sediment transport by waves. Proc., 15th Coastal Eng. Conf., ASCE, Reston, VA, 1093-1112.

Menter, F. R., 1994. Two-equation eddy-viscosity turbulence models for engineering applications. AIAA J., 32(8), 269-289.

Menter, F. R., Kuntz, M., Langtry, R., 2003. Ten years of industrial experience with the SST turbulence model. Turbulence, Heat and Mass Transfer 4, Begell House, Danbury, CT., 625-632.

Oldenziel, D. M., Brink, W. E., 1974. Influence of suction and blowing on entrainment of sand particles. J. Hydr. Div., ASCE, 100(7), 935-949.

Pan, D., Wang, L., Pan, C., and Hu, J., 2007. Experimental investigation on the wave-induced 
pore pressure around shallowly embedded pipelines. Acta Oceanologica Sinica, 26, 125 135.

Ramakrishna Rao, A., Nagaraj, S., 1999. Stability and mobility of sand-bed channels affected by seepage. J. Irrigat. Drainage Eng., 125(6), 370-379.

Richardson, J. R., Abt, S. R., Richardson, E. V., 1985. Inflow seepage influence on straight alluvial channels. J. Hydraul.Eng., ASCE, 111(8), 1133-1147.

Seed, H. B., Rahman, M. S., 1978. Wave-induced pore pressure in relation to ocean floor stability of cohesionless soils. Mar. Geotec., 3(2), 123-150.

Shields, A., 1936. Application of similitude mechanics and research on turbulence to bed load movement. Mitteilungender Preussischer Versuchsanstalt fur Wasserbau und Schiffbau 26 (in German).

Sumer, B. M., Jensen, H. R., Fredsøe, J., 1988. Effect of lee-wake on scour below pipelines in current. J. Waterw. Port Coast. Ocean Eng., ASCE, 114(5), 599-614.

Sumer, B. M., Jensen, B. L., Fredsøe, J., 1991. Effect of plane boundary on oscillatory flow around a circlar cylinder. J. Fluid Mech., 225, 271-300.

Sumer, B. M., Fredsøe, J., 2002. The mechanics of scour in the marine environment. World Scientific, Singapore.

Sumer B. M., Chua L. H. C., Cheng N. -S. and Fredsøe J., 2003. Influenceof turbulence on bed load sediment transport. J. Hydraul.Eng., ASCE, 129(8), 585-596.

Sumer, B. M., Fredsøe, J., 2006. Hydrodynamics around cylindrical structures. World Scientific, Singapore.

Sumer, B. M., Sen, M. B., Karagali, I., Ceren, B., Fredsøe, J., Sottile, M., Zilioli, L. and Fuhrman, D.R., 2011. Flow and Sediment transport induced by a plunging solitary wave. J. Geophys. Res., 116, C01008, 1-15.

Sumer, B. M., Kirca, V. S. O., Fredsøe, J., 2012. Experimental validation of a mathematical model for seabed liquefaction under waves. Int. J. Offshore and Polar Engineering, 22(2), $133-141$.

Sumer, B. M., Guner, A. A., Hansen, N. M., Fuhrman, D. R. ,Fredsøe, J., 2013. Laboratory investigations of flow and sediment transport induced by plunging regular waves. J. Geopgysical Res., 118(11), 6161-6182.

Soulsby, R., 1997. Dynamics of marine sands. Thomas Telford Publications, Kent. Tsai, C. P., and Lee, T. L. 1995. Standing wave induced pore pressures in a porous seabed. Ocean Eng., 22(6), 505-517.

Valyrakis, M., Diplas, P., Dancey, C. L., Greer, K., Celik, A. O., 2010. The role of 
instantaneous force magnitude and duration of particle entrainment. J. Geophysical Res., 115(F2), 2003-2012.

Whitehouse, R. J. S, Hardisty, J., 1988. Experimental assessment of two theories for the effect of bedslope on the threshold of bedload transport. Mar. Geol., 79, 135-139.

Yamamoto, T., Koning, H., Sellmeijer, H., Hijum, E. V., 1978. On the response of a poroelastic bed to water waves. J. Fluid Mech., 87(1), 193-206.

Zen, K., Yamazaki, H., 1990. Mechanism of wave-induced liquefaction and densification in seabed. Soil Found., 30(4), 90-104.

Zhao, H. Y., Jeng, D. S., Guo, Z., Zhang J. S., 2014. Two-dimensional model for pore pressure accumulations in the vicinity of a buried pipeline. J. Offshore Mech. Arct., 136, 042001, 1-15. 


\section{Figures}

Fig. 1 The sketch of entire numerical model

Fig. 2 Comparison of the present model with experimental data (Jensen, 1987) for mean horizontal flow velocity $U_{\mathrm{x}} / U_{0}$ : (a) $x=1.0 D$; (b) $x=1.5 D$; (c) $x=2.5 D$; (d) $x=4.5 D$; (e) $x=8.5 D$; (f) $x=16.5 D$

Fig. 3 Comparison of the present model with experimental data (Jensen, 1987) for mean vertical flow velocity $U_{\mathrm{z}} / U_{0}$ : (a) $x=1.0 D$; (b) $x=1.5 \mathrm{D}$; (c) $x=2.5 \mathrm{D}$; (d) $x=4.5 \mathrm{D}$; (e) $x=8.5 \mathrm{D}$; (f) $x=16.5 \mathrm{D}$

Fig. 4 Comparison of the present model with experimental data (Jensen, 1987) for horizontal velocity fluctuation $\Delta U_{\mathrm{x}} / U_{0}$ : (a) $x=1.0 D$; (b) $x=1.5 D$; (c) $x=2.5 D$; (d) $x=4.5 D$; (e) $x=8.5 D$; (f) $x=16.5 D$

Fig. 5 Comparison of the present model with experimental data (Jensen, 1987) for vertical velocity fluctuation $\Delta U_{\mathrm{z}} / U_{0}$ : (a) $x=1.0 D$; (b) $x=1.5 \mathrm{D}$; (c) $x=2.5 \mathrm{D}$; (d) $x=4.5 \mathrm{D}$; (e) $x=8.5 \mathrm{D}$; (f) $x=16.5 \mathrm{D}$

Fig. 6 Drag and lift coefficients versus dimensionless period $t U_{0} / D$

Fig. 7 Flow pattern during typical vortex shedding period: (a) $t=t_{0}+0.0$; (b) $t=t_{0}+T / 4$; (c) $t=t_{0}+2 \times T / 4$; (d) $t=t_{0}+3 \times T / 4$

Fig. 8 Pressure coefficient along the seabed surface

Fig. 9 Oscillatory pore-pressure in a typical vortex shedding period: (a) $t=t_{0}+0.0$; (b) $t=t_{0}+T / 6$; (c) $t=t_{0}+2 \times T / 6 ;$ (d) $t=t_{0}+3 \times T / 6 ;$ (e) $t=t_{0}+4 \times T / 6 ;$ (f) $t=t_{0}+5 \times T / 6$

Fig. 10 Accumulation of the residual pore-pressure within the seabed: (a) $t=50 \mathrm{~s}$; (b) $t=100 \mathrm{~s}$; (c) $t=150 \mathrm{~s}$; (d) $t=250 \mathrm{~s} ;$ (e) $t=500 \mathrm{~s}$; (f) $t=900 \mathrm{~s}$

Fig. 11 Forces exerting on a bed particle: (a) Plain view; (b) Side view

Fig. 12 Time-averaged, maximum and minimum bed shear stresses

Fig. 13 Comparisons of critical Shields number with original and modified Shields numbers

Fig. 14 Distributions of two scaling factors along the seabed surface: (a) Variations of oscillatory factor $\tilde{e}$; (b) Variations of residual factor $\bar{e}$

Fig. 15 Effect of the saturation degree $S_{r}$ on maximum oscillatory factor $\tilde{e}$

Fig. 16 Effect of the saturation degree $S_{r}$ on residual factor $\bar{e}$

Fig. 17 Effect of the permeability coefficient $k_{s}$ on maximum oscillatory factor $\tilde{e}$

Fig. 18 Effect of the permeability coefficient $k_{s}$ on residual factor $\bar{e}$

Fig. 19 Effect of the soil shear modulus $G_{s}$ on maximum oscillatory factor $\tilde{e}$

Fig. 20 Effect of the soil shear modulus $G_{s}$ on residual factor $\bar{e}$ 
Fig. 21 Positions of particles resting on the bed surface (Liu and Chiew, 2012): (a) Position 1; (b) Position 2; (c) Position 3; (d) Position 4 


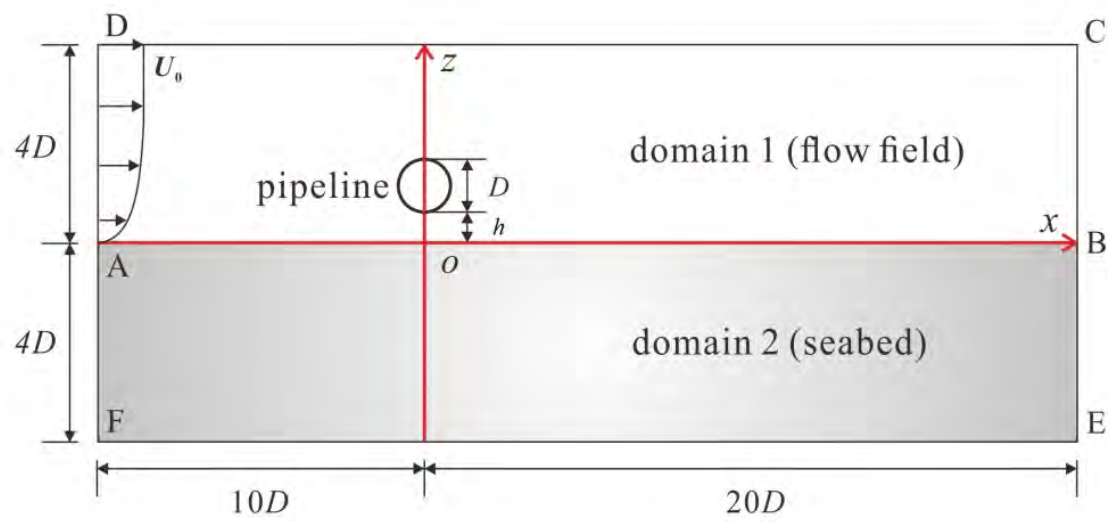

Fig. 1 The sketch of whole numerical model 

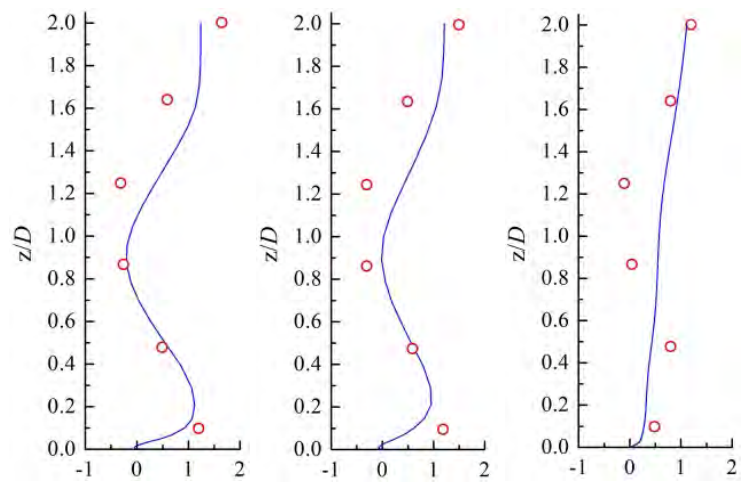

(a) $x=1.0 \mathrm{D}$

\begin{abstract}
(b) $x=1.5 \mathrm{D}$
\end{abstract}
(c) $x=2.5 \mathrm{D}$

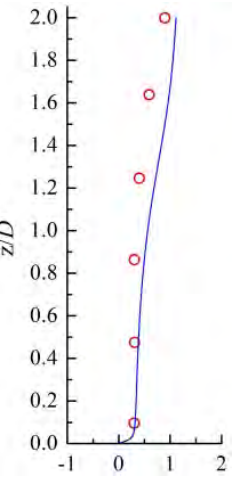

(d) $x=4.5 \mathrm{D}$
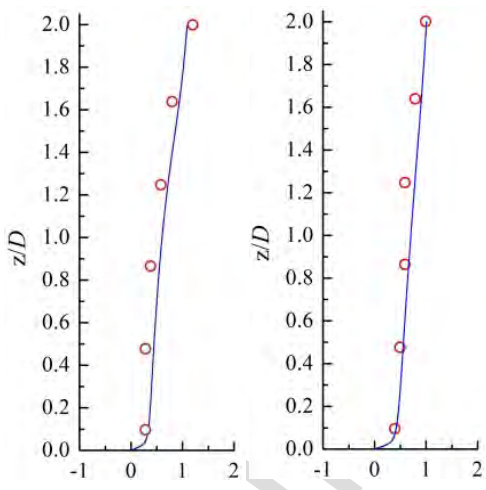

(e) $x=8.5 \mathrm{D}$

(f) $x=16.5 D$

O Mean velocity measured by Jensen (1987)

Fig. 2 Comparison of the present model with experimental data (Jensen, 1987) for mean horizontal flow velocity $U_{\mathrm{x}} / U_{0}$ 


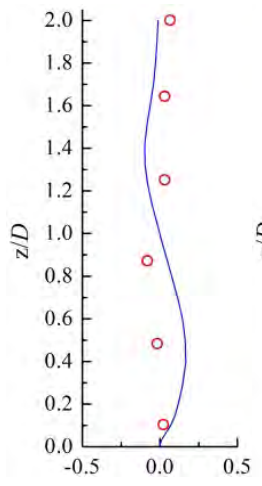

(a) $x=1.0 \mathrm{D}$
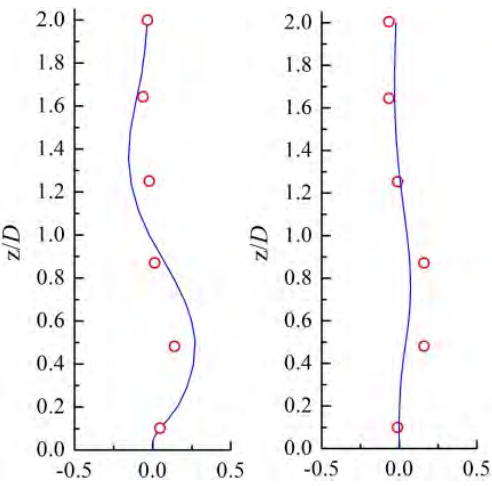

(b) $x=1.5 D$

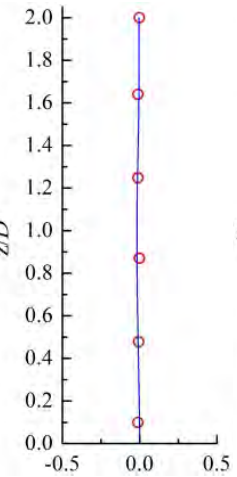

(d) $x=4.5 D$
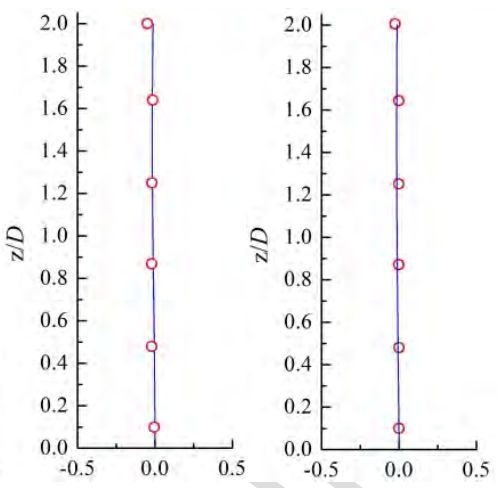

(e) $x=8.5 \mathrm{D}$

(f) $x=16.5 \mathrm{D}$

O Mean velocity measured by Jensen (1987)

- Mean velocity calculated by SST

Fig. 3 Comparison of the present model with experimental data (Jensen, 1987) for mean vertical flow velocity $U_{z} / U_{0}$ 


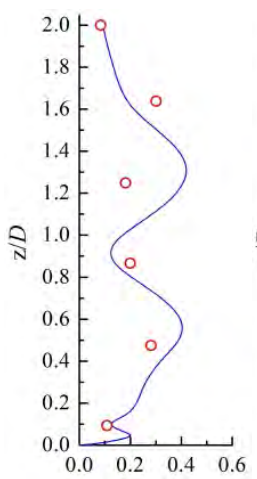

(a) $x=1.0 \mathrm{D}$

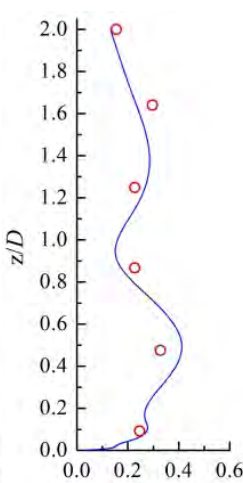

(b) $x=1.5 \mathrm{D}$

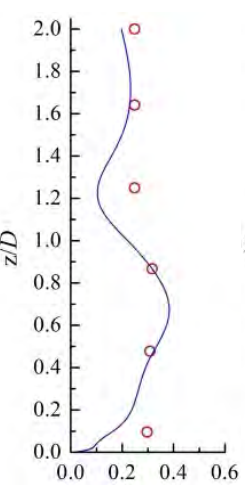

(c) $x=2.5 \mathrm{D}$

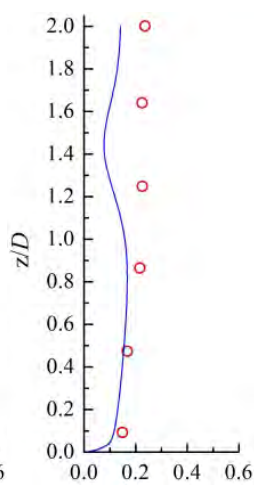

(d) $x=4.5 \mathrm{D}$
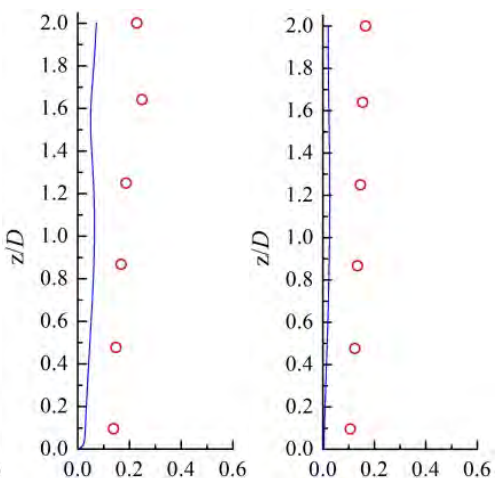

(e) $x=8.5 \mathrm{D}$

(f) $x=16.5 \mathrm{D}$

O Velocity fluctuations measured by Jensen (1987)

Fig. 4 Comparison of the present model with experimental data (Jensen, 1987) for horizontal velocity fluctuation $\Delta U_{\mathrm{x}} / U_{0}$ 


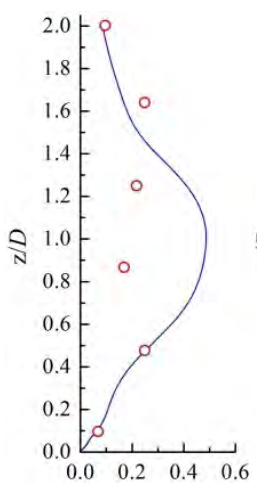

(a) $x=1.0 \mathrm{D}$

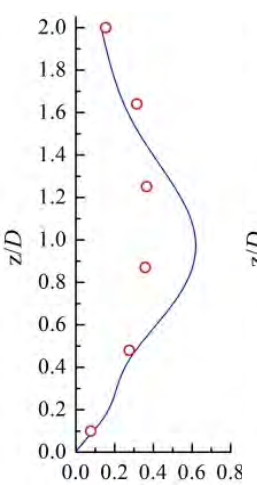

(b) $x=1.5 \mathrm{D}$

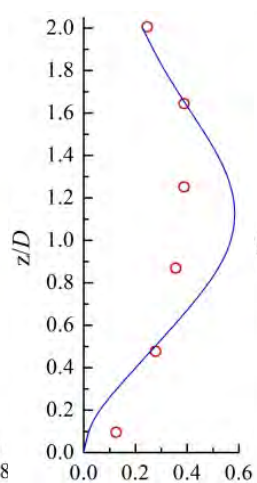

(c) $x=2.5 \mathrm{D}$

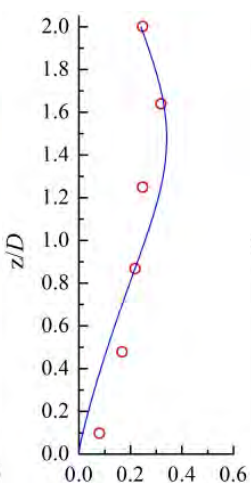

(d) $x=4.5 \mathrm{D}$
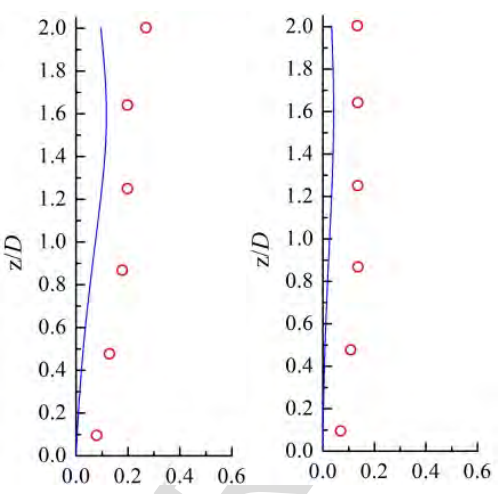

(e) $x=8.5 \mathrm{D}$

(f) $x=16.5 \mathrm{D}$

O Velocity fluctuations measured by Jensen (1987)

- Velocity fluctuations calculated by SST

Fig. 5 Comparison of the present model with experimental data (Jensen, 1987) for vertical velocity fluctuation $\Delta U_{\mathrm{z}} / U_{0}$ 


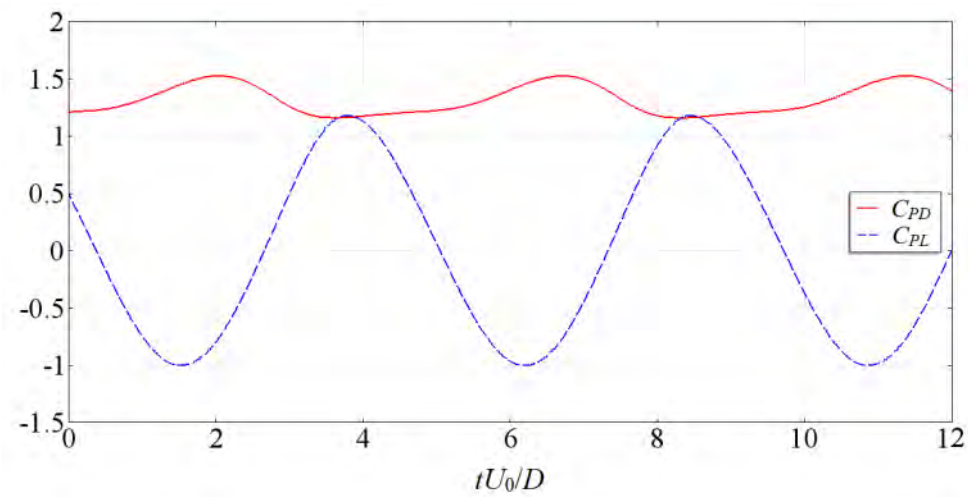

Fig. 6 Drag and lift coefficients versus dimensionless period $t U_{0} / D$ 


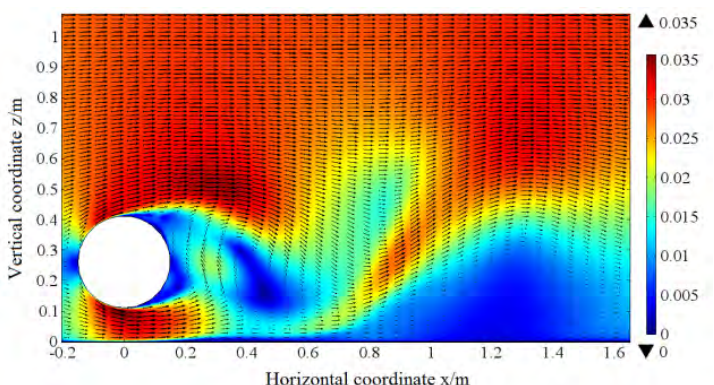

(a) $t=t_{0}+0.0$

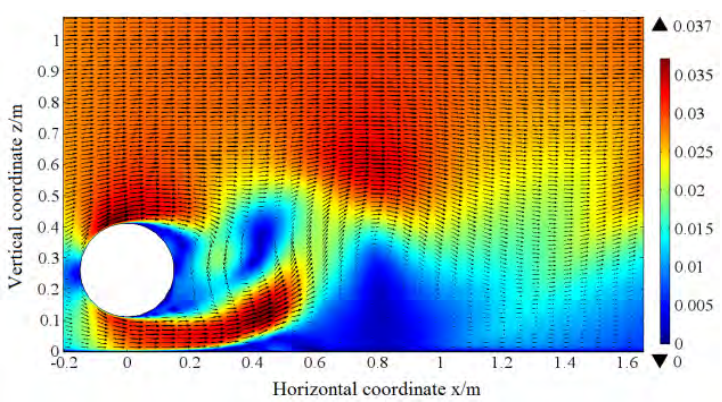

(c) $t=t_{0}+2 \times T / 4$

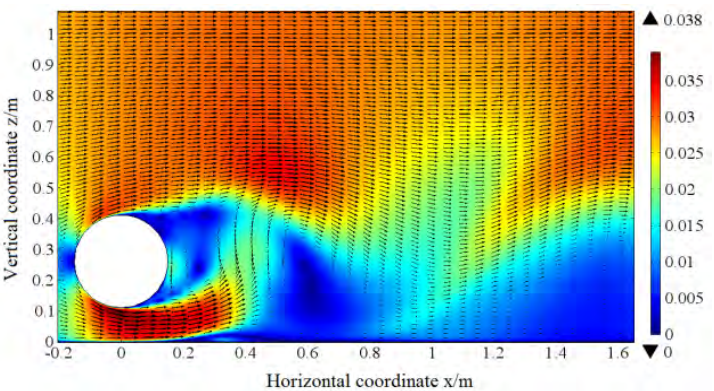

(b) $t=t_{0}+T / 4$

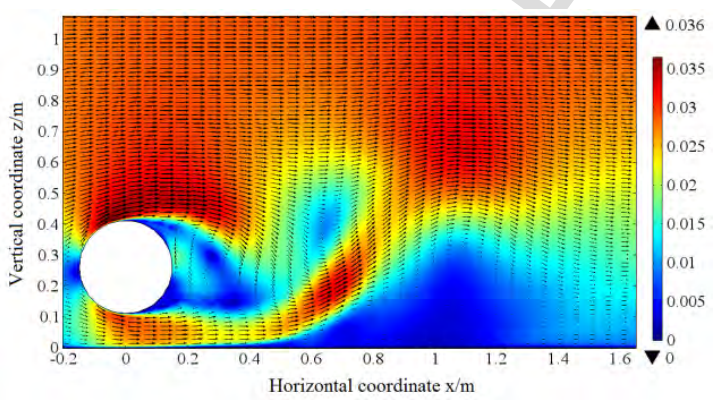

(d) $t=t_{0}+3 \times T / 4$

Fig. 7 Flow pattern during typical vortex shedding period 


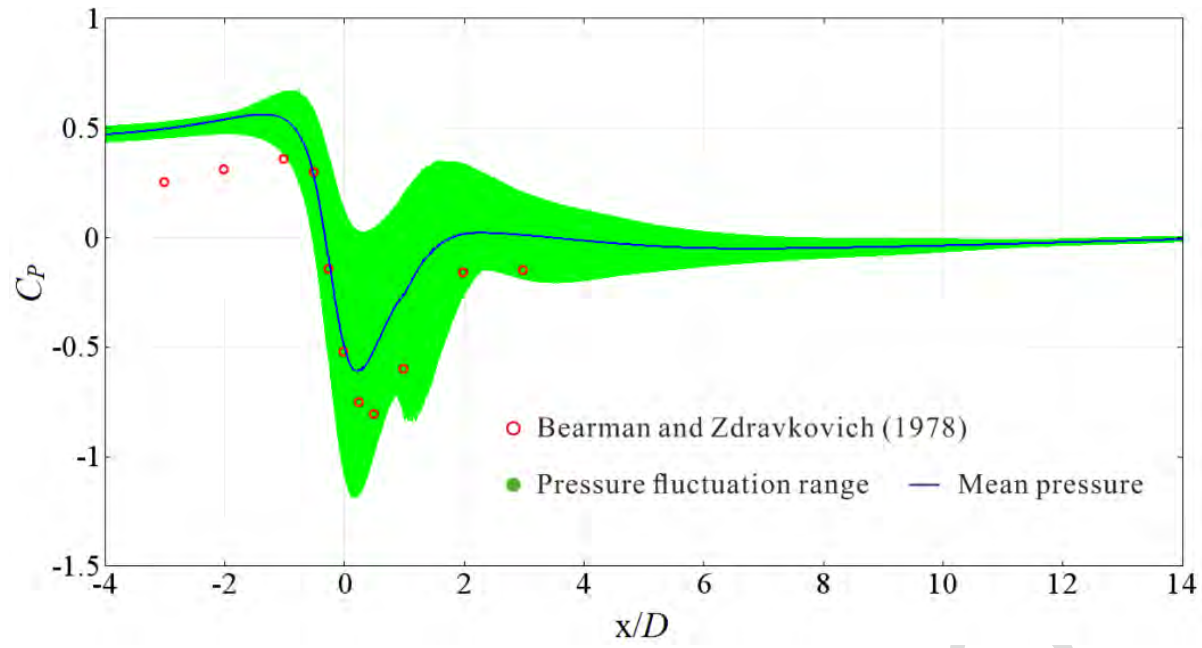

Fig. 8 Pressure coefficient along the seabed surface 


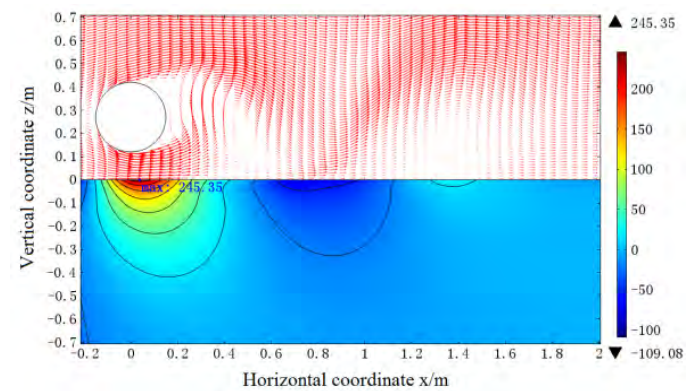

(a) $t=t_{0}+0.0$

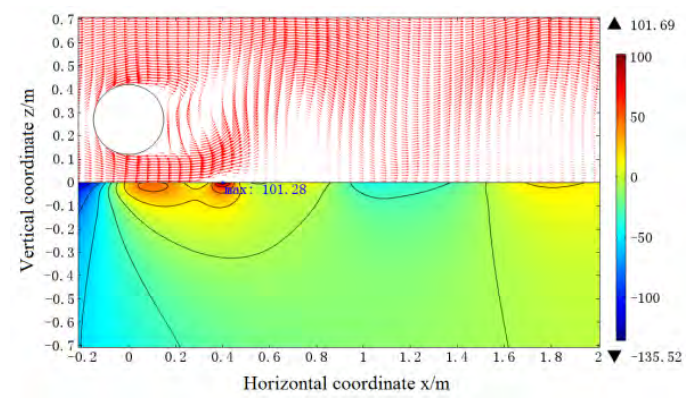

(c) $t=t_{0}+2 \times T / 6$

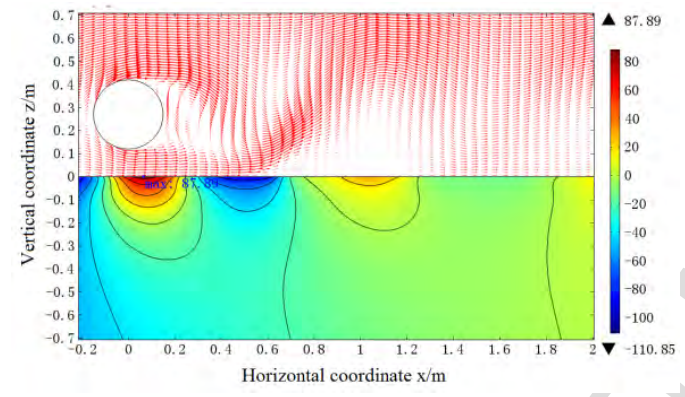

(e) $t=t_{0}+4 \times T / 6$

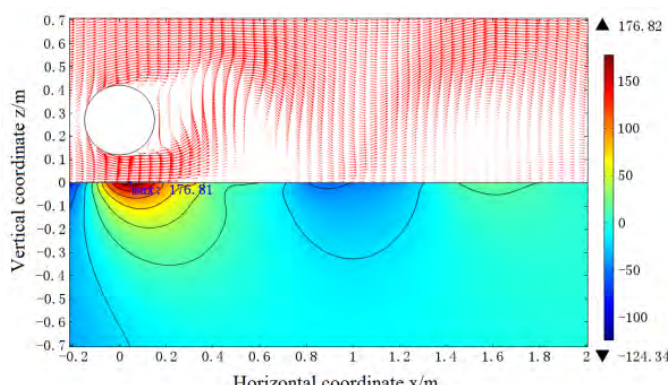

(b) $t=t_{0}+T / 6$

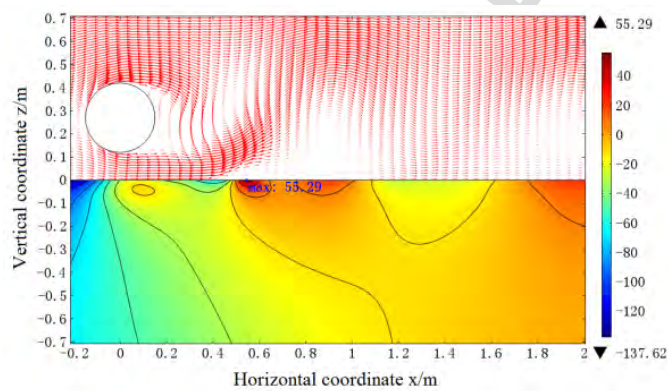

(d) $t=t_{0}+3 \times T / 6$

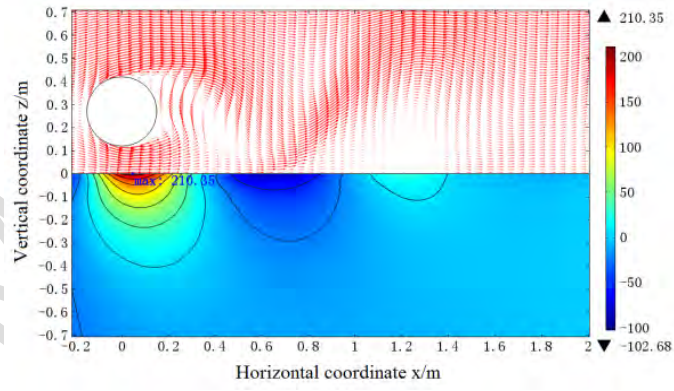

(f) $t=t_{0}+5 \times T / 6$

Fig. 9 Oscillatory pore-pressure in a typical vortex shedding period 


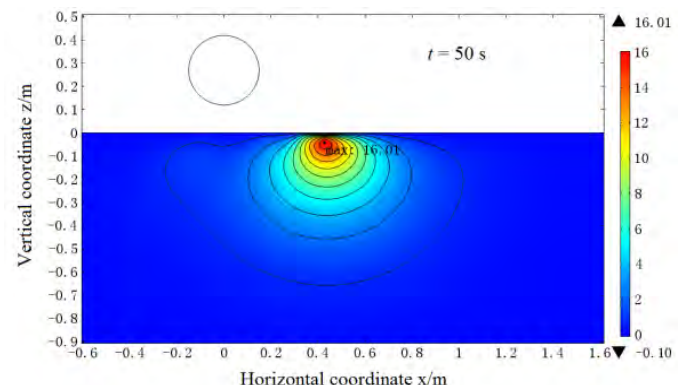

(a) $t=50 \mathrm{~s}$

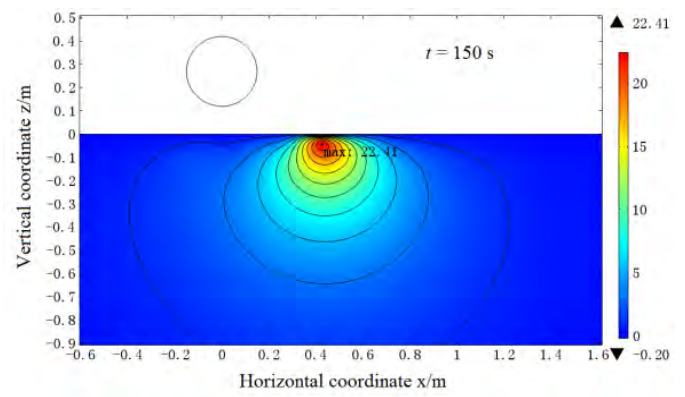

(c) $t=150 \mathrm{~s}$

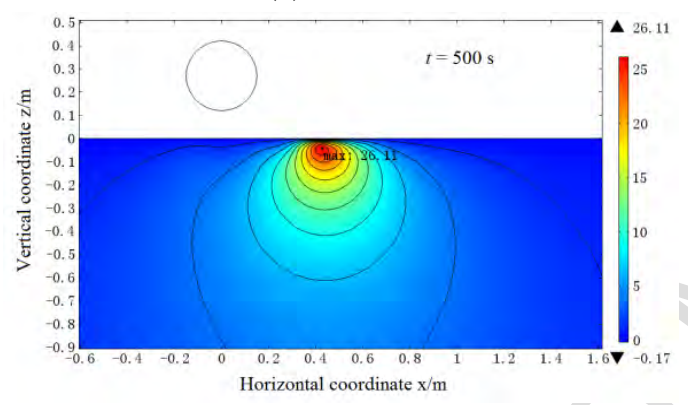

(e) $t=500 \mathrm{~s}$

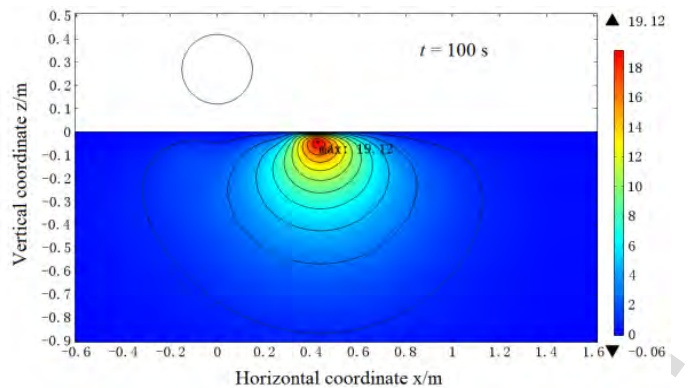

(b) $t=100 \mathrm{~s}$

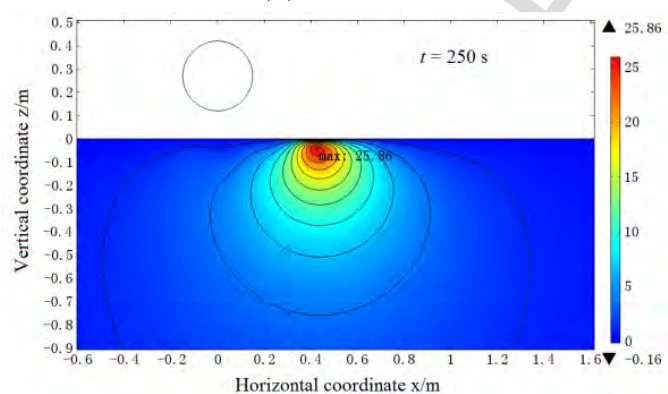

(d) $t=250 \mathrm{~s}$

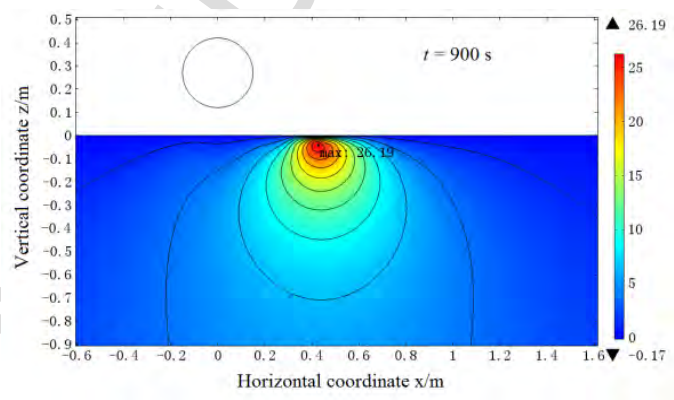

(f) $t=900 \mathrm{~s}$

Fig. 10 Accumulation of the residual pore-pressure within the seabed 
$\Rightarrow$ flow direction

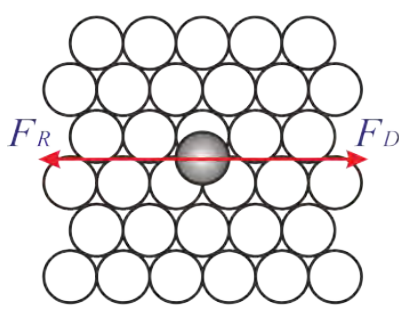

(a) Plain view
$\Rightarrow$ flow direction

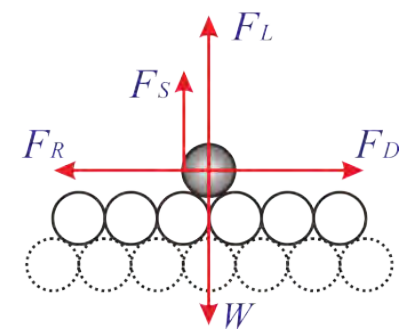

(b) Side view

Fig. 11 Forces exerting on a bed particle 


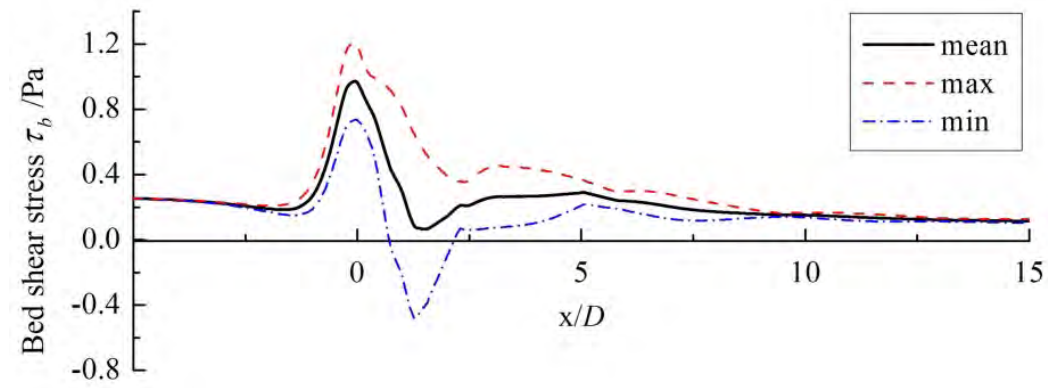

Fig.12 Time-averaged, maximum and minimum bed shear stresses 


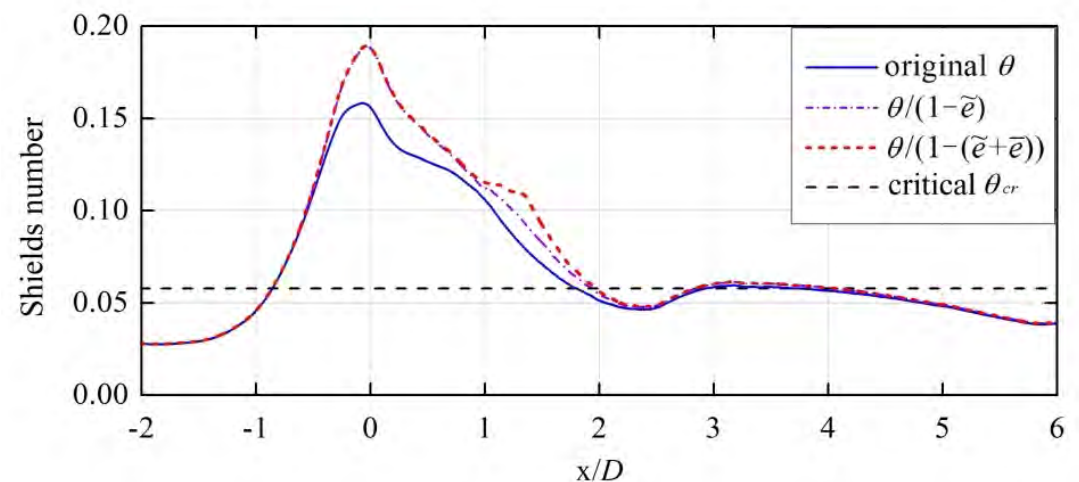

Fig. 13 Comparisons of critical Shields number with original and modified Shields numbers 


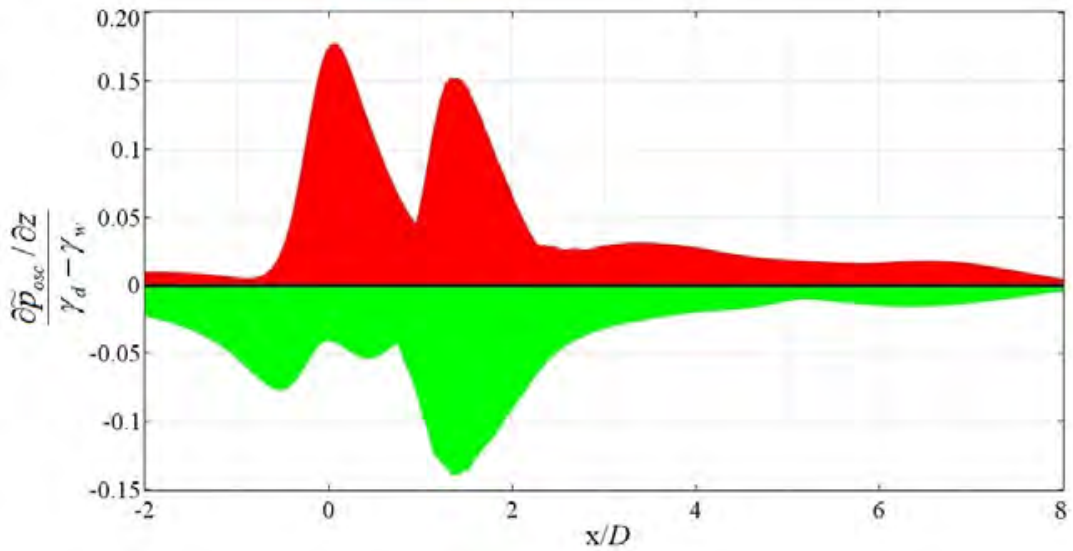

(a) Variations of oscillatory factor $\tilde{\boldsymbol{e}}$

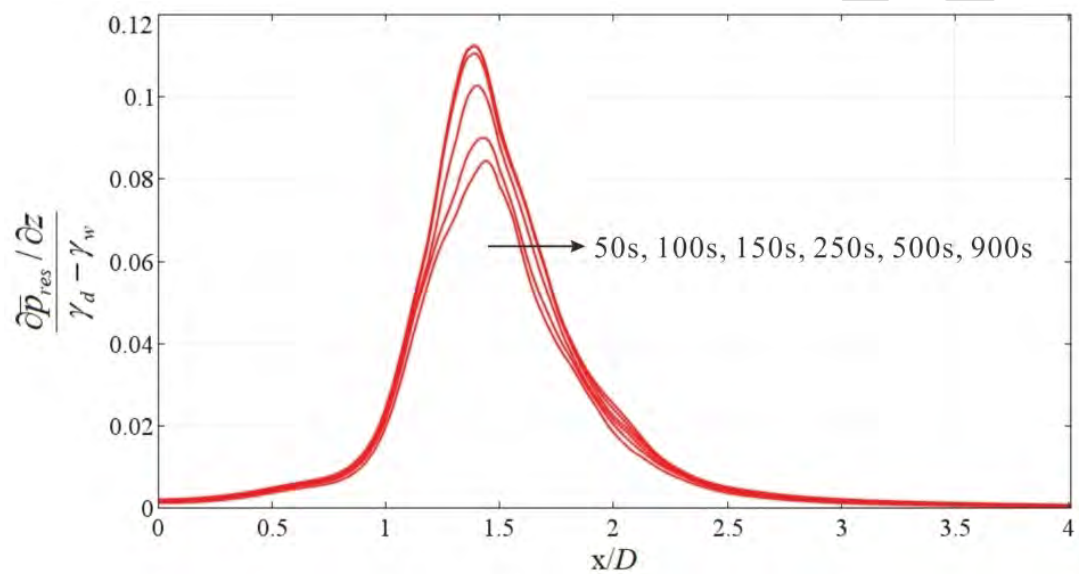

(b) Variations of residual factor $\bar{e}$

Fig. 14 Distributions of two scaling factors along the seabed surface 


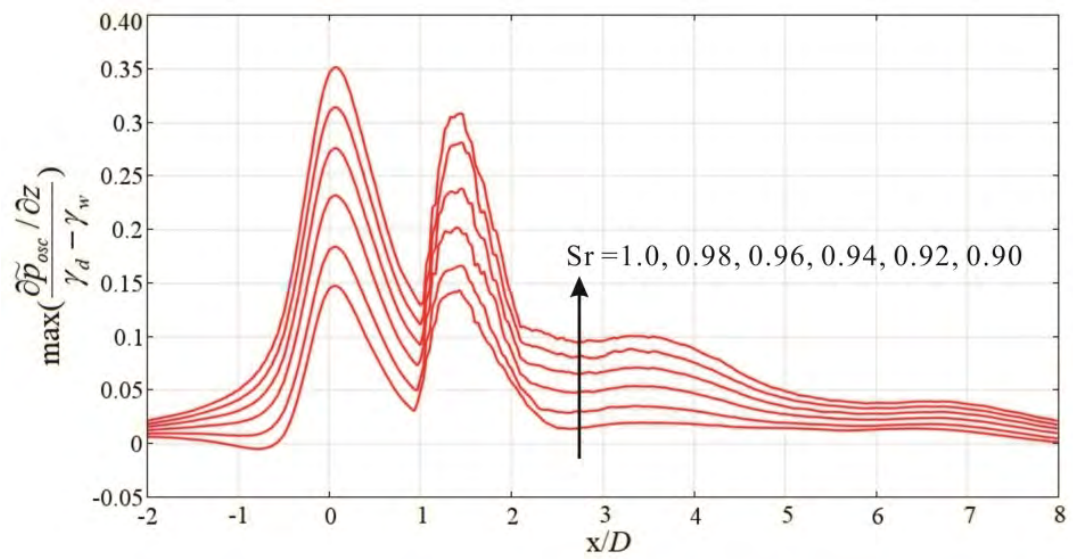

Fig. 15 Effect of the saturation degree $S_{r}$ on maximum oscillatory factor $\tilde{\boldsymbol{e}}$ 


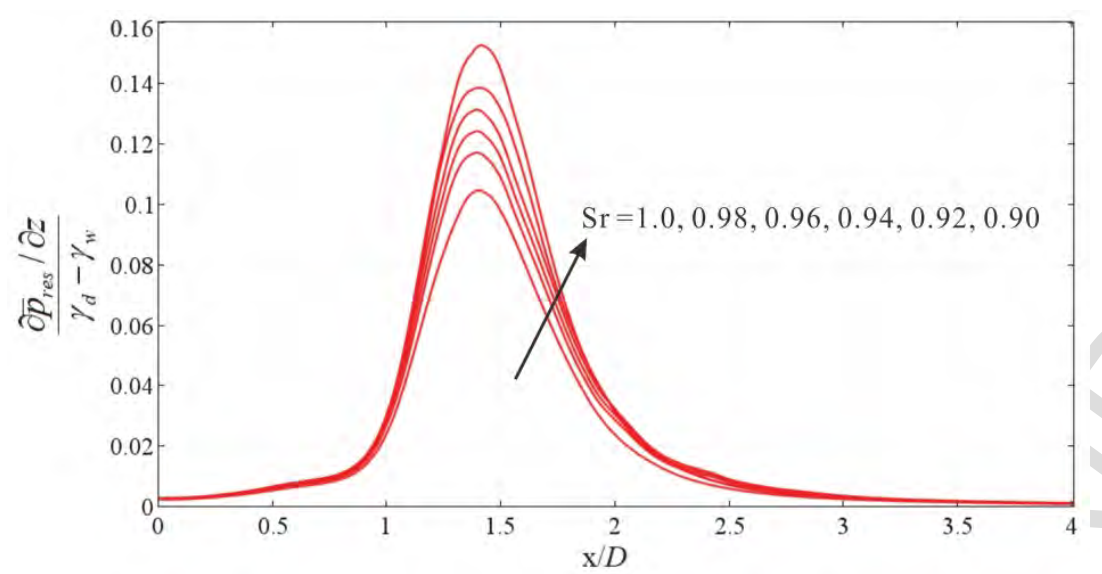

Fig. 16 Effect of the saturation degree $S_{r}$ on residual factor $\bar{e}$ 


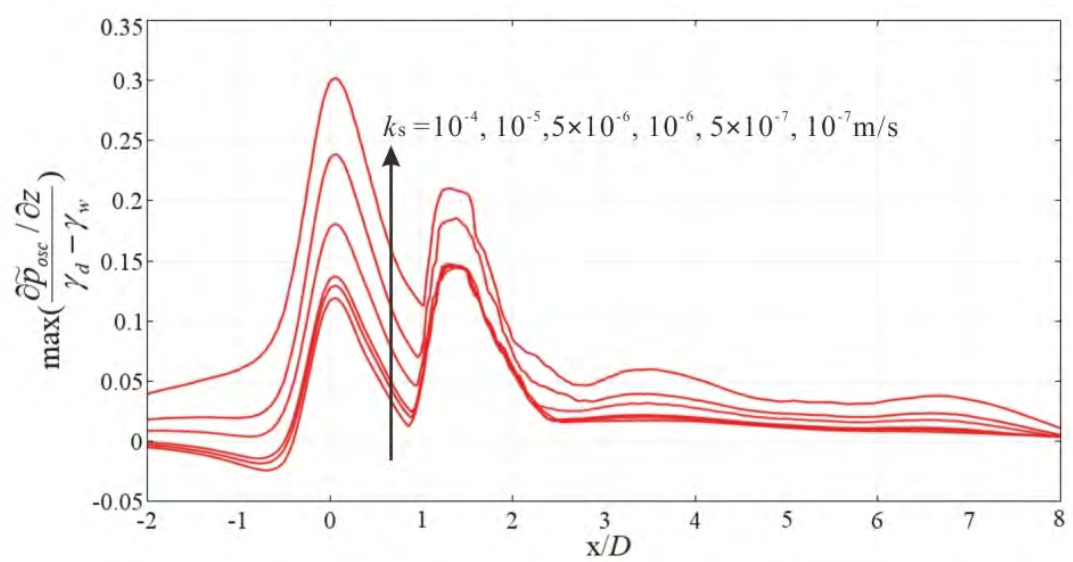

Fig. 17 Effect of the permeability coefficient $k_{s}$ on maximum oscillatory factor $\widetilde{e}$ 


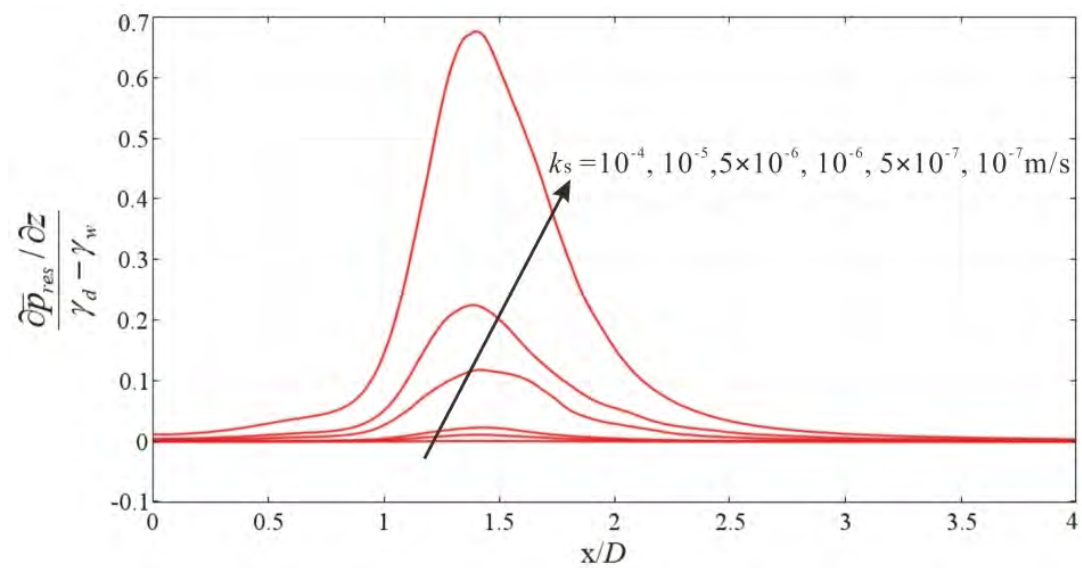

Fig. 18 Effect of the permeability coefficient $k_{s}$ on residual factor $\bar{e}$ 


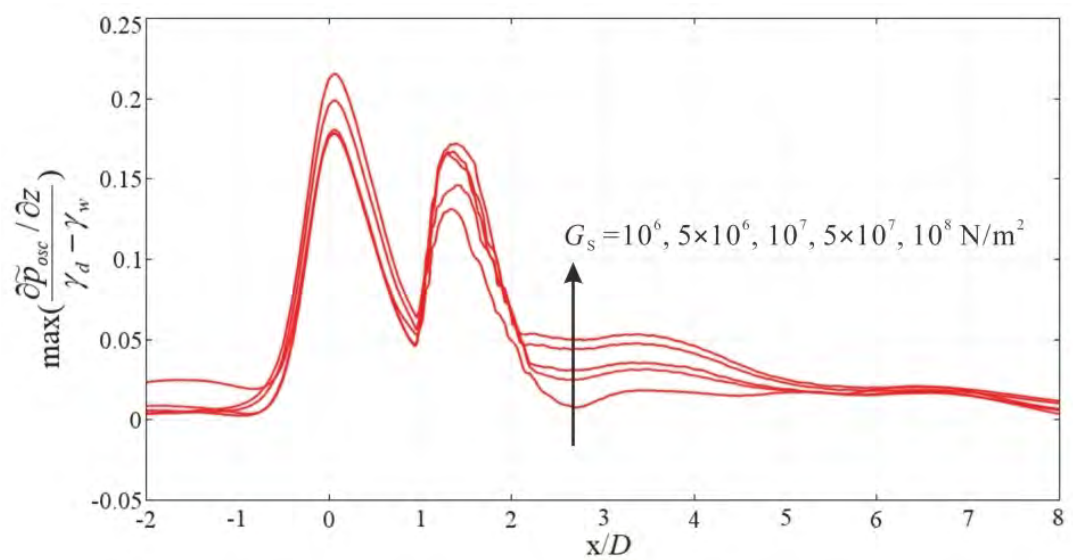

Fig. 19 Effect of the soil shear modulus $G_{s}$ on maximum oscillatory factor $\tilde{e}$ 


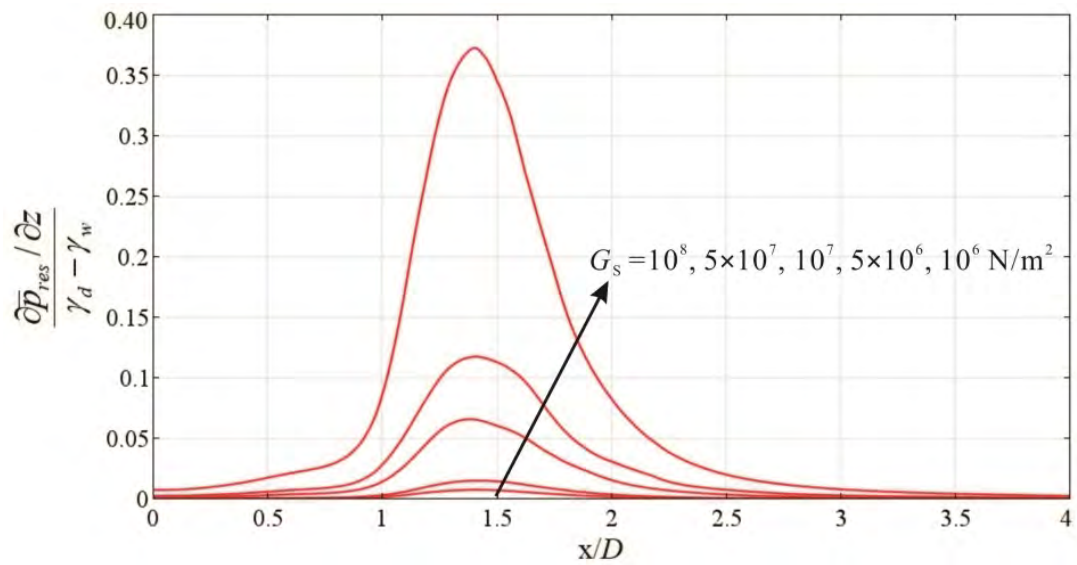

Fig. 20 Effect of the soil shear modulus $G_{s}$ on residual factor $\bar{e}$ 


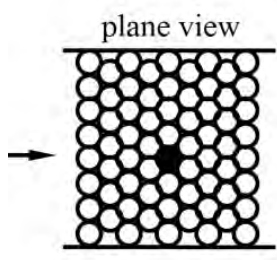

000000000

side view

(a) Position 1
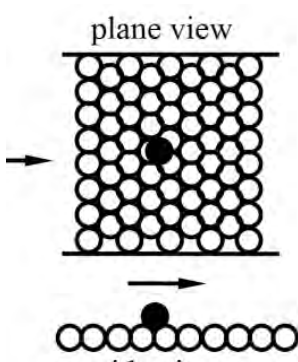

side view

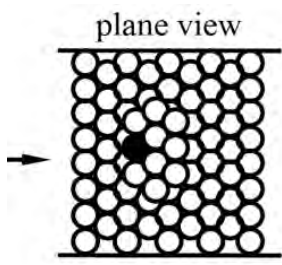



side view
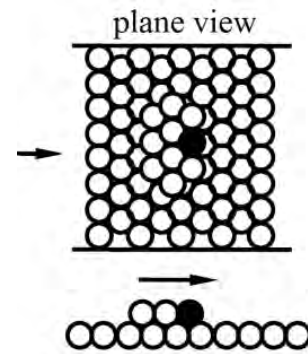

side view

(d) Position 4

Fig. 21 Positions of particles resting on the bed surface (Liu and Chiew, 2012) 


\section{Research Highlights}

(a) Propose an integrated numerical model to couple the turbulent flow and porous seabed models

(b) Study the excess pore-pressure responses in the seabed beneath the suspended pipeline

(c) Quantify the effect of seepage flow on the mobility of bed particles

(d) Study the effects of seabed parameters on the flow-induced seepage force. 


\section{Tables}

Table 1 Seabed soil parameters for the numerical model 
Table 1 Seabed soil parameters for the numerical model

\begin{tabular}{ll}
\hline Seabed soil parameters & values \\
\hline median grain size $d_{50}$ & $0.36 \mathrm{~mm}$ \\
total unit weight of soil $\gamma_{s}$ & $2000 \mathrm{~kg} / \mathrm{m}^{3}$ \\
unit weight of pore water $\gamma_{w}$ & $1030 \mathrm{~kg} / \mathrm{m}^{3}$ \\
permeability coefficient $k_{s}$ & $1 \times 10^{-6} \mathrm{~m} / \mathrm{s}$ \\
degree of seabed saturation $S_{r}$ & 0.985 \\
earth pressure coefficient $K_{0}$ & 0.42 \\
soil porosity $n_{s}$ & 0.44 \\
void ratio $r_{s}$ & 0.8 \\
maximum void ratio $r_{\max }$ & 1.2 \\
minimum void ratio $r_{\min }$ & 0.4 \\
relative density of soil $D_{r}$ & 0.5 \\
Poisson's ratio $v_{s}$ & 0.33 \\
shear modulus of soil $G_{s}$ & $5 \times 10^{6} \mathrm{~N} / \mathrm{m}^{2}$ \\
\hline
\end{tabular}




\section{Accepted Manuscript}

Effect of Seepage Flow on Sediment Incipient Motion around a Free Spanning Pipeline

Zhen Guo, Dong-Sheng Jeng, Hongyi Zhao, Wei Guo, Lizhong Wang

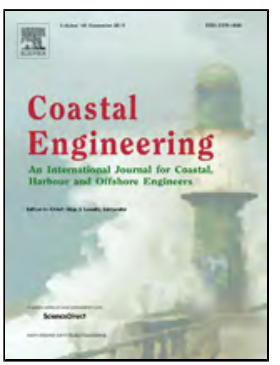

PII: $\quad$ S0378-3839(18)30529-5

DOI: $\quad$ 10.1016/j.coastaleng.2018.10.012

Reference: $\quad$ CENG 3440

To appear in: $\quad$ Coastal Engineering

Received Date: $\quad 28$ August 2014

Accepted Date: $\quad 27$ October 2018

Please cite this article as: Zhen Guo, Dong-Sheng Jeng, Hongyi Zhao, Wei Guo, Lizhong Wang, Effect of Seepage Flow on Sediment Incipient Motion around a Free Spanning Pipeline, Coastal Engineering (2018), doi: 10.1016/j.coastaleng.2018.10.012

This is a PDF file of an unedited manuscript that has been accepted for publication. As a service to our customers we are providing this early version of the manuscript. The manuscript will undergo copyediting, typesetting, and review of the resulting proof before it is published in its final form. Please note that during the production process errors may be discovered which could affect the content, and all legal disclaimers that apply to the journal pertain. 